MODELING, IDENTIFICATION AND CONTROL, 2004, vol. 25, No. 1, 3-27

doi:10.4173/mic.2004.1.1

\title{
A Nonlinear Ship Manoeuvering Model: Identification and adaptive control with experiments for a model ship
}

\author{
ROGER SKJETNE*, ØYVIND N. SMOGELI†† and \\ THOR I. FOSSEN*\$
}

Keywords: Ship control; manoeuvering; nonlinear ship model; system identification, robust adaptive control; experimental testing

\begin{abstract}
Complete nonlinear dynamic manoeuvering models of ships, with numerical values, are hard to find in the literature. This paper presents a modeling, identification, and control design where the objective is to manoeuver a ship along desired paths at different velocities. Material from a variety of references have been used to describe the ship model, its difficulties, limitations, and possible simplifications for the purpose of automatic control design. The numerical values of the parameters in the model is identified in towing tests and adaptive manoeuvering experiments for a small ship in a marine control laboratory.
\end{abstract}

\section{Introduction}

Model-based control for steering and positioning of ships has become state-ofthe-art since LQG and similar state-space techniques were applied in the 1960s. For a rigid-body the dynamic equations of motion are divided into two distinctive parts: kinematics, which is the study of motion without reference to the forces that cause motion, and kinetics, which relates the action of forces on bodies to their resulting motions (Meriam \& Kraige, 1993). The rigid-body and hydrodynamic equations of motion for a ship are in reality given by a set of (very complicated) differential equations describing the 6 degrees-of-freedom (6 DOF); surge, sway, and heave for translation, and roll, pitch, and yaw for rotation. The models used to represent the physics of the real world, however, vary as much as the underlying control objectives vary. Roughly divided these control objectives are either slow speed positioning or high speed steering. The first is called dynamical positioning (DP) and includes station keeping, position mooring, and slow speed reference tracking. For DP the 6 DOF model is reduced to a simpler 3 DOF model that is linear in the kinetic part. Such applications with references are thoroughly described by Strand (1999) and Lindegaard (2003). High speed steering, on the other hand, includes automatic course control, high speed position tracking, and path following; see for instance Holzhüter (1997), Lefeber et al. (2003) and Fossen et al. (2003). For these applications, Coriolis and centripetal forces together with nonlinear viscous effects become increasingly important and therefore make the kinetic part nonlinear. By port-starboard symmetry, the longitudinal (surge) dynamics are essentially decoupled from the lateral (steering; sway-yaw) dynamics and can therefore be controlled independently by

* Department of Engineering Cybernetics, Norwegian University of Science and Technology (NTNU), NO-7491 Trondheim, Norway. E-mails: skjetne@ieee.org, tif@itk.ntnu.no

$\dagger$ Department of Marine Technology, Norwegian University of Science and Technology (NTNU), NO-7491 Trondheim, Norway. E-mail: oyvind.smogeli@marin.ntnu.no

t Centre for Ships and Ocean Structures (CESOS), Norwegian University of Science and Technology (NTNU), NO-7491 Trondheim, Norway. 
forward propulsion. Moreover, for cruising at a nearly constant surge speed and only considering first order approximations of the viscous damping, a linear parametrically varying approximation of the steering dynamics is applicable. The origin of these types of models are traced back to Davidson \& Schiff (1946), while Nomoto et al. (1957) gave an equivalent representation. See Clarke (2003) for a historical background and Fossen (2002) for a complete reference on these original models and their later derivations.

The contribution of this paper is a 3 DOF nonlinear manoeuvering model for a ship. This model can be simplified further to either a 3 DOF model for DP, a steering model according to Davidson and Schiff or Nomoto, or it can be used as is for nonlinear control design. Furthermore, system identification procedures for a model ship called CyberShip II (CS2) in a towing tank facility have produced numerical values for nearly all the hydrodynamic coefficients. To find the other values, an adaptive manoeuvering control law was implemented for CS2, and free-running manoeuvering experiments were performed. The adaptive parameter estimates in these experiments then give approximate values for the other hydrodynamic coefficients.

Notation: Total time derivatives of $x(t)$ are denoted $\dot{x}, \ddot{x}, x^{(3)}, \ldots, x^{(n)}$, while a superscript denotes partial differentiation:

$$
\alpha^{t}(x, \theta, t):=\frac{\partial \alpha}{\partial t}, \quad \alpha^{x^{2}}(x, \theta, t):=\frac{\partial^{2} \alpha}{\partial x^{2}} \quad \text { and } \quad \alpha^{\theta^{n}}(x, \theta, t):=\frac{\partial^{n} \alpha}{\partial \theta^{n}}, \text { etc. }
$$

The Euclidean vector norm is $|x|:=\left(x^{\top} x\right)^{1 / 2}$ which reduces to the absolute value for a scalar.

\section{The 3 DOF ship manoeuvering model}

Ship dynamics are described by 6 degrees-of-freedom (6 DOF) differential equations of motion. The modes are $(x, y, z)$, referred to as surge, sway, and heave, describing the position in three-dimensional space, and $(\phi, \theta, \psi)$, called roll, pitch, and yaw, describing the orientation of the ship. Assuming that the ship is longitudinally and laterally metacentrically stable with small amplitudes $\phi=\theta=\dot{\phi}=\dot{\theta} \approx 0$, one can discard the dynamics of roll and pitch. Likewise, since the ship is floating with $z \approx 0$ in mean, one can discard the heave dynamics. The resulting model for the purpose of manoeuvering the ship in the horizontal plane becomes a 3 DOF model. Let an inertial frame be approximated by the earth-fixed reference frame $\{\mathrm{e}\}$ called NED (North-East-Down) and let another coordinate frame $\{b\}$ be attached to the ship as seen in Figure 1. The states of the vessel can then be taken as $\eta=[x, y, \psi]^{\top}$ and $v=[u, v, r]^{\top}$ where $(x, y)$ is the Cartesian position, $\psi$ is the heading (yaw) angle, $(u, v)$ are the body-fixed linear velocities (surge and sway), and $r$ is the yaw rate.

\subsection{Rigid-body dynamics}

The earth-fixed velocity vector is related to the body-fixed velocity vector through the kinematic relationship

$$
\dot{\eta}=R(\psi) v
$$




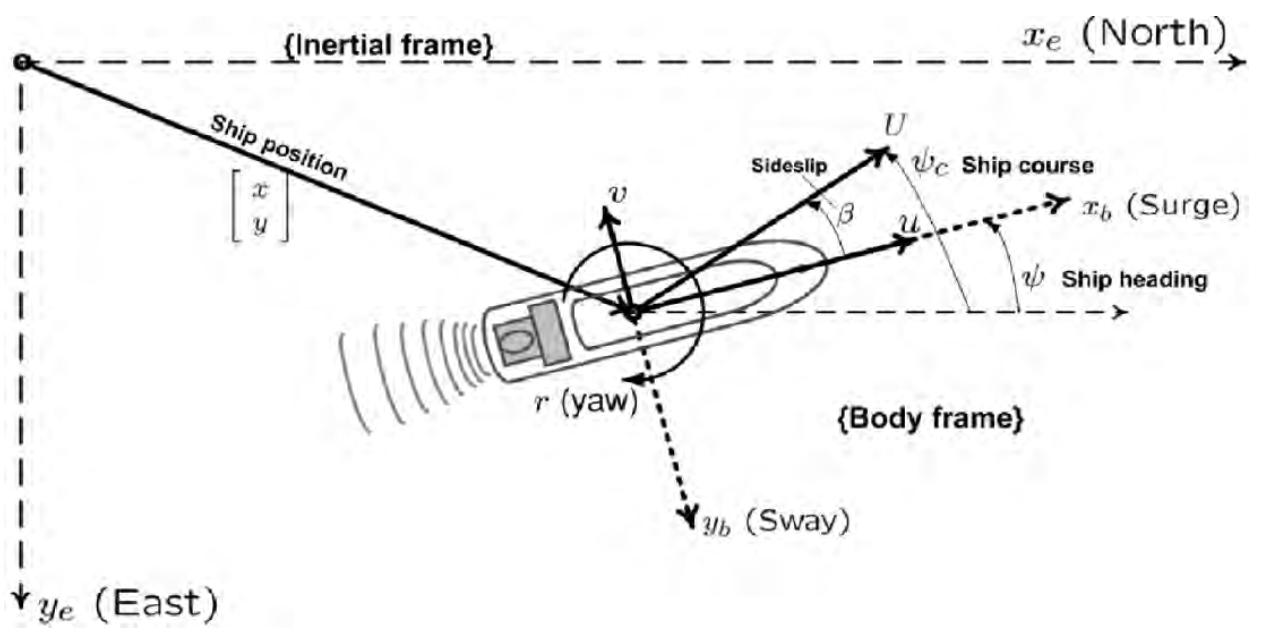

Figure 1. Figure showing the inertial earth-fixed frame and the body-fixed frame for a ship with the earth-fixed position $(x, y)$, the heading $\psi$, and the corresponding body-fixed velocities $(u, v)$ and rotation rate $r$.

where

$$
R(\psi)=\left[\begin{array}{ccc}
\cos \psi & -\sin \psi & 0 \\
\sin \psi & \cos \psi & 0 \\
0 & 0 & 1
\end{array}\right]
$$

is a rotation matrix. It has the properties that $R(\psi)^{\top} R(\psi)=I,\|R(\psi)\|=1$ for all $\psi$, and $(d / d t)\{R(\psi)\}=\dot{\psi} R(\psi) S$ where

$$
S=\left[\begin{array}{rrr}
0 & -1 & 0 \\
1 & 0 & 0 \\
0 & 0 & 0
\end{array}\right]=-S^{\top}
$$

is skew-symmetric. By Newton's second law it is shown in Fossen (2002) that the rigid body equations of motion can be written

$$
M_{R B} \dot{v}+C_{R B}(v) v=\tau_{R B}
$$

where $M_{R B}$ is the rigid-body system inertia matrix, $C_{R B}(v)$ is the corresponding matrix of Coriolis and centripetal terms, and $\tau_{R B}=[X, Y, N]^{\top}$ is a generalized vector of external forces $(X, Y)$ and moment $N$. Let the origin ' $\mathrm{O}$ ' of the body frame be taken as the geometric center point $(\mathrm{CP})$ in the ship structure. Under the assumption that the ship is port-starboard symmetric, the center-of-gravity (CG) will be located a distance $x_{g}$ along the body $x_{b}$-axis. In this case, $M_{R B}$ takes the form

$$
M_{R B}=\left[\begin{array}{ccc}
m & 0 & 0 \\
0 & m & m x_{g} \\
0 & m x_{g} & I_{z}
\end{array}\right]
$$


where $m$ is the mass of the ship and $I_{z}$ is the moment of inertia about the $z_{b}$-axis (that is, yaw rotation). Several representations for the Coriolis matrix are possible. Based on Theorem 3.2 in Fossen (2002), we choose the skew-symmetric representation

$$
C_{R B}(v)=\left[\begin{array}{ccc}
0 & 0 & -m\left(x_{g} r+v\right) \\
0 & 0 & m u \\
m\left(x_{g} r+v\right) & -m u & 0
\end{array}\right]
$$

The force and moment vector $\tau_{R B}$ is given by the superposition of actuator forces and moments $\tau=\left[\tau_{u}, \tau_{v}, \tau_{r}\right]^{\top}$, hydrodynamic effects $\tau_{H}$, and exogenous disturbances $w(t)$ due to, for instance, waves and wind forces (Sørensen et al., 1996). The forces and moments in $\tau_{R B}$ are all expressed with reference to the center point (CP) such that the full set of dynamical equations is given in the body-fixed reference frame.

\subsection{Hydrodynamic forces and moments}

The vector $\tau_{H}$ is the result of several hydrodynamic phenomena, some not yet fully understood. For an ideal fluid, some of these components are added mass, radiation-induced potential damping, and restoring forces. For the 3 DOF states considered here, restoring forces are only important in case of mooring which is not in the scope of this paper. In addition to potential damping there are also other damping effects such as skin friction, wave drift damping, and damping due to vortex shedding (Faltinsen, 1990).

Due to currents in the ocean fluid, the velocity $v$ is different than the relative velocity $v_{r}$ between the ship hull and the fluid. The hydrodynamic forces and moments depend on this relative velocity. For a nonrotational current with fixed speed $V_{c}$ and angle $\beta_{c}$ in the earth-fixed frame, the current velocity is given by

$$
v_{c}:=\left[\begin{array}{c}
V_{c} \cos \beta_{c} \\
V_{c} \sin \beta_{c} \\
0
\end{array}\right]
$$

Normally $V_{c}$ and $\beta_{c}$ should be modeled as stochastic processes. However, in the deterministic setting of this paper we simply assume that $\dot{V}_{c}=\dot{\beta}_{c}=0$. In the body-frame this gives the current component $v_{c}:=R(\psi)^{\top} v_{c}$ and the relative velocity $v_{r}:=v-v_{c}=$ $\left[u_{r}, v_{r}, r\right]^{\top}$. With these definitions it is common (Sørensen, 2002) to model the hydrodynamic effects as

$$
\tau_{H}=-M_{A} \dot{v}_{r}-C_{A}\left(v_{r}\right) v_{r}-d\left(v_{r}\right)
$$

where $M_{A}$ accounts for added mass, $C_{A}\left(v_{r}\right)$ accounts for the corresponding added Coriolis and centripetal terms, and $d\left(v_{r}\right)$ sums up the damping effects. By the notation of The Society of Naval Architects and Marine Engineers (1950) the matrix $M_{A}$ is given by

$$
M_{A}=\left[\begin{array}{ccc}
X_{\dot{u}} & 0 & 0 \\
0 & -Y_{\dot{v}} & -Y_{\dot{r}} \\
0 & -N_{\dot{v}} & -N_{\dot{r}}
\end{array}\right]
$$


where the assumption of port-starboard symmetry again is applied. For zero relative velocity, $v_{r}=0$, zero frequency of motion due to water surface effects, and assuming an ideal fluid, the added mass matrix is constant and $M_{A}=M_{A}^{\top}>0$. However, under non-ideal conditions with waves and high velocity, $M_{A}=M_{A}\left(\omega_{e}\right) \neq M_{A}\left(\omega_{e}\right)^{\top}$ where $\omega_{e}$ is the frequency of encounter given by

$$
\omega_{e}=\left|\omega_{0}-\frac{\omega_{0}^{2}}{g} U \cos \beta\right|
$$

Here $\omega_{0}$ is the dominating wave frequency, $g$ is the acceleration of gravity, $U=\sqrt{u^{2}+v^{2}}$ is the total ship speed, and $\beta$ is the angle of encounter defined by $\beta=0^{\circ}$ for following sea. For control design it is common to assume that $M_{A}=\lim _{\omega_{e} \rightarrow 0} M_{A}\left(\omega_{e}\right)$ is constant and strictly positive.

Since $M_{A}$ is not necessarily symmetric, Theorem 3.2 in Fossen (2002) is not directly applicable to find $C_{A}\left(v_{r}\right)$. To solve this obstacle, we observe that this theorem is deduced from the kinetic energy $T=\frac{1}{2} v^{\top} M v$. A modification for the added mass kinetic energy is

$$
T_{A}=\frac{1}{2} v_{r}^{\top} M_{A} v_{r}=\frac{1}{4} v_{r}\left(M_{A}+M_{A}^{\top}\right) v_{r}=\frac{1}{2} v_{r} \bar{M}_{A} v_{r}
$$

where $\bar{M}_{A}:=\frac{1}{2}\left(M_{A}+M_{A}^{\top}\right)=\bar{M}_{A}^{\top}$. This means that $C_{A}\left(v_{r}\right)$, for a nonsymmetric $M_{A}$, is derived from Theorem 3.2 of Fossen (2002) using $\bar{M}_{A}$ instead of $M_{A}$, and this gives

$$
C_{A}\left(v_{r}\right)=\left[\begin{array}{ccc}
0 & 0 & Y_{\dot{v}} v_{r}+\frac{1}{2}\left(N_{\dot{v}}+Y_{\dot{r}}\right) r \\
0 & 0 & -X_{\dot{u}} u_{r} \\
-Y_{\dot{v}} v_{r}-\frac{1}{2}\left(N_{\dot{v}}+Y_{\dot{r}}\right) r & X_{\dot{u}} u_{r} & 0
\end{array}\right]
$$

The most uncertain component in the hydrodynamic model (6) is the damping vector $d\left(v_{r}\right)$, to which many hydrodynamic phenomena contribute. Let $d\left(v_{r}\right)=\left[X_{D}\left(v_{r}\right)\right.$, $\left.Y_{D}\left(v_{r}\right), N_{D}\left(v_{r}\right)\right]^{\top}$. For a constant cruise speed $v_{r}=v_{0} \approx\left[u_{0}, 0,0\right]^{\top}$ one can fit the damping forces and moments at $v_{0}$ to the linear functions

$$
\begin{gathered}
X_{D}\left(v_{r}\right)=-X_{u}\left(u_{r}-u_{0}\right)-X_{v} v_{r}-X_{r} r \\
Y_{D}\left(v_{r}\right)=-Y_{u}\left(u_{r}-u_{0}\right)-Y_{v} v_{r}-Y_{r} r \\
N_{D}\left(v_{r}\right)=-N_{u}\left(u_{r}-u_{0}\right)-N_{v} v_{r}-N_{r} r
\end{gathered}
$$

where the hydrodynamic coefficients $\left\{X_{(\cdot)}, Y_{(\cdot)}, N_{(\cdot)}\right\}$ are called hydrodynamic derivatives because they are the partial derivatives of the forces and moment with respect to the corresponding velocities, for instance,

$$
Y_{r}:=\frac{\partial Y_{D}\left(v_{r}\right)}{\partial r}
$$

Seeking in this paper a more globally valid model of the damping effects, we consider a nonlinear representation. Abkowitz (1964) proposed using a truncated Taylor series expansion of $d\left(v_{r}\right)$. Since in general $d\left(v_{r}\right)$ is dissipative for both positive and negative relative velocities, it must be an odd function, and, hence, only odd terms in the Taylor expansion are required. Using first and third order terms only, and assuming port-starboard symmetry, this gives 


$$
\begin{aligned}
& X_{D}\left(v_{r}\right)=-X_{u} u_{r}-X_{u u u} u_{r}^{3} \\
& Y_{D}\left(v_{r}\right)=-Y_{v} v_{r}-Y_{r} r-Y_{v v v} v_{r}^{3}-Y_{v v r} v_{r}^{2} r-Y_{v r r} v_{r} r^{2}-Y_{r r r} r^{3} \\
& N_{D}\left(v_{r}\right)=-N_{v} v_{r}-N_{r} r-N_{v v v} v_{r}^{3}-N_{v v r} v_{r}^{2} r-N_{v r r} v_{r} r^{2}-N_{r r r} r^{3}
\end{aligned}
$$

which is valid for all feasible velocities. Fedyaevsky and Sobolev (1963) and later Norrbin (1970) gave another nonlinear representation

$$
\begin{aligned}
& X_{D}\left(v_{r}\right)=-X_{u} u_{r}-X_{|u| u}\left|u_{r}\right| u_{r} \\
& Y_{D}\left(v_{r}\right)=-Y_{v} v_{r}-Y_{r} r-Y_{|v| v}\left|v_{r}\right| v_{r}-Y_{|v| r}\left|v_{r}\right| r-Y_{|r| v}|r| v_{r}-Y_{|r| r \mid}|r| r \\
& N_{D}\left(v_{r}\right)=-N_{v} v_{r}-N_{r} r-N_{|v| v}\left|v_{r}\right| v_{r}-N_{|v| r}\left|v_{r}\right| r-N_{|r| v}|r| v_{r}-N_{|r| r}|r| r
\end{aligned}
$$

called the second order modulus model. These functions are not continuously differentiable, and strictly speaking they therefore cannot represent the physical system. However, experiments have shown that they match the damping effects quite accurately and are therefore often used. Based on the experimental data presented in the next section and curve fitting, we choose in this paper the damping model

$$
d\left(v_{r}\right)=D_{L} v_{r}+D_{N L}\left(v_{r}\right) v_{r}=: D\left(v_{r}\right) v_{r}
$$

where

$$
\begin{aligned}
D_{L} & :=\left[\begin{array}{ccc}
-X_{u} & 0 & 0 \\
0 & -Y_{v} & -Y_{r} \\
0 & -N_{v} & -N_{r}
\end{array}\right] \\
D_{N L}\left(v_{r}\right): & =\left[\begin{array}{ccc}
-X_{|u| u \mid}\left|u_{r}\right|-X_{u u u} u_{r}^{2} & 0 & 0 \\
0 & -Y_{|v| v}\left|v_{r}\right|-Y_{|r| v}|r| & -Y_{|v| r}\left|v_{r}\right|-Y_{|r| r|r|}|r| \\
0 & -N_{|v| v}\left|v_{r}\right|-N_{|r| v}|r| & -N_{|v| r}\left|v_{r}\right|-N_{|r| r} \mid r
\end{array}\right]
\end{aligned}
$$

which essentially is the second order modulus model with an extra third order term in surge. The reason for picking this model was that it gave the best fit to the experimental data.

With $\tau_{R B}=\tau+\tau_{H}+w(t)$ the kinetic equation of motion (2) becomes

$$
M_{R B} \dot{v}+M_{A} \dot{v}_{r}+C_{R B}(v) v+C_{A}\left(v_{r}\right) v_{r}+D\left(v_{r}\right) v_{r}=\tau+w(t)
$$

where

$$
\begin{aligned}
& v_{r}=v-R(\psi)^{\top} v_{c} \\
& \dot{v}_{r}=\dot{v}-r S^{\top} R(\psi)^{\top} v_{c}
\end{aligned}
$$

For the kinetic model (14) one must decide upon using either the relative velocity $v_{r}$ or the inertial velocity $v$ as the velocity state. There are different practices in the literature, and the current velocity $v_{c}$ must in either case be measured or somehow estimated to account for it in equation (14). A simplifying technique was applied by Fossen \& Strand (1999) who used $v$ as the velocity state and assumed that the dynamics related to the current $v_{c}$ (and other unmodeled dynamics) are captured by a slowly varying bias $b$ in the earth frame. This gives the simplified model

$$
M \dot{v}+C(v) v+D(v) v=\tau+R(\psi)^{\top} b+w(t)
$$


where $M:=M_{R B}+M_{A}$ and $C(v):=C_{R B}(v)+C_{A}(v)$. The alternative, applied among others by Holzhüter (1997), is to use $v_{r}$ as the state, but in this case the kinematic relationship (1) must be rewritten as

$$
\dot{\eta}=R(\psi) v_{r}+v_{c}
$$

which means that $v_{c}$ enters both the kinematic and kinetic equations of motion.

For simulator design, a model according to equation (14) or more advanced should be used. For control design, on the other hand, experience shows that equations (1) and (15) are adequate provided some type of integral action is used in the controller to compensate for the bias $b$; see for instance Skjetne \& Fossen (2004).

\subsection{Simplified models}

For special applications, simpler models than equation (15) can be used. For instance, for DP a linearization of equation (15) around $v=0$ yields

$$
M \dot{v}+D_{L} v=\tau+R(\psi)^{\top} b+w(t)
$$

where the Coriolis and nonlinear damping terms were eliminated. Note that the curve-fitted coefficients in $D_{L}$ for DP will be different from those fitted to the nonlinear (globally valid) model equation (13); see next section.

Another special application is steering a ship at (nearly) constant surge speed. Separating the surge dynamics from the steering dynamics, using equation (10), and assuming port-starboard symmetry and $v_{c} \equiv 0$, we get a manoeuvering model consisting of the surge dynamics

$$
\left(m-X_{\dot{u}}\right) \dot{u}-X_{u}\left(u-u_{0}\right)-\left(m-Y_{\dot{v}}\right) v r-\left(m x_{g}-\frac{1}{2} N_{\dot{v}}-\frac{1}{2} Y_{\dot{r}}\right) r^{2}=\tau_{u}
$$

and the sway-yaw (steering) dynamics

$$
\begin{aligned}
& {\left[\begin{array}{cc}
m-Y_{\dot{v}} & m x_{g}-Y_{\dot{r}} \\
m x_{g}-N_{\dot{v}} & I_{z}-N_{\dot{r}}
\end{array}\right]\left[\begin{array}{c}
\dot{v} \\
\dot{r}
\end{array}\right]} \\
& +\left[\begin{array}{cc}
-Y_{v} & -Y_{r}+\left(m-X_{\dot{u}}\right) u \\
-N_{v}+\left(X_{\dot{u}}-Y_{\dot{v}}\right) u & -N_{r}+\left(m x_{g}-\frac{1}{2} N_{\dot{v}}-\frac{1}{2} Y_{\dot{r}}\right) u
\end{array}\right]\left[\begin{array}{l}
v \\
r
\end{array}\right]=\left[\begin{array}{c}
\tau_{v} \\
\tau_{r}
\end{array}\right]
\end{aligned}
$$

For each fixed surge speed $u=u_{0}$, the steering dynamics become linear. Hence, treating $u$ as a parameter, equation (19) is a linear parametrically varying (LPV) model of the form of Davidson \& Schiff (1946). This can be further related to a Nomoto model as described by Clarke (2003). For conventional ships, the inputs are usually linearly related to the rudder angle $\delta$ as $\tau_{v}=-Y_{\delta} \delta$ and $\tau_{r}=-N_{\delta} \delta$. As a result, linear design techniques as gain scheduling or similar can be applied to solve a steering task.

\subsection{Actuator forces}

The actuator forces and moments are generated by a set of thrusters with revolutions per second $n=\left[n_{1}, n_{2}, \ldots, n_{p_{1}}\right]^{\top} \in \mathbb{R}^{p_{1}}$ and a set of control surfaces (or 


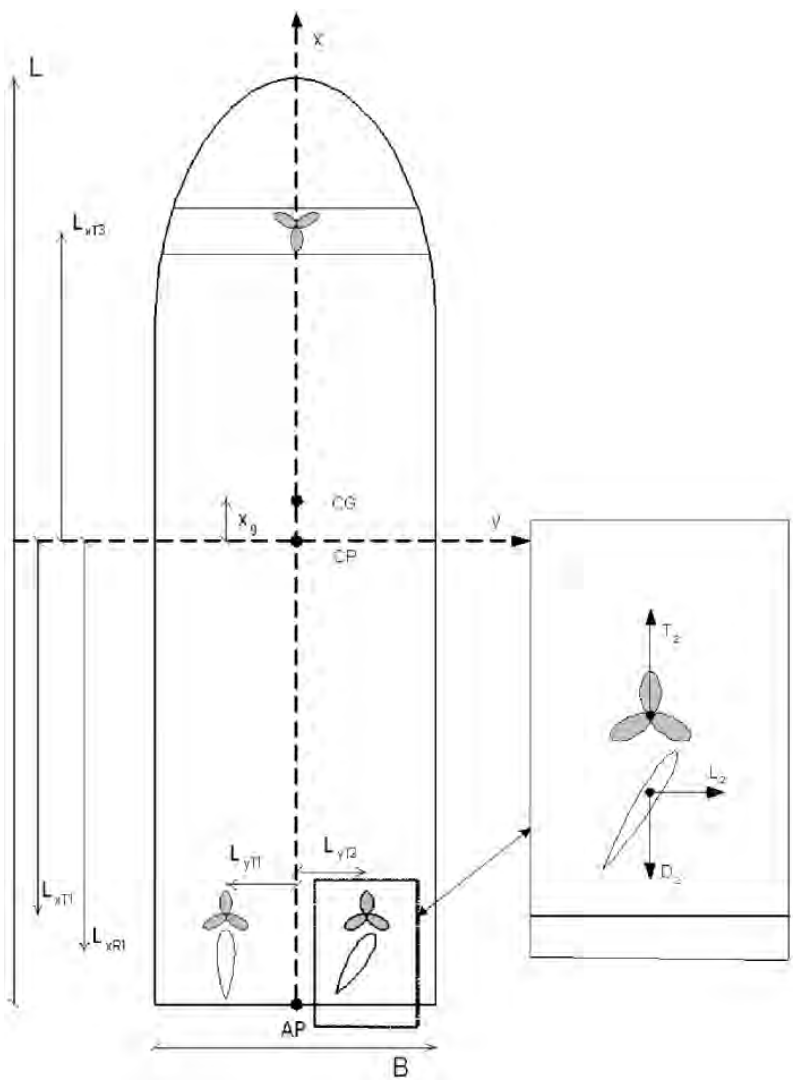

Figure 2. Actuator configuration of CyberShip II.

propeller blade pitch) with angles $\delta=\left[\delta_{1}, \delta_{2}, \ldots, \delta^{p_{2}}\right]^{\top} \in \mathbb{R}^{p_{2}}$. They are related to the input vector $\tau$ through the mapping

$$
\tau=B f_{c}\left(v_{r}, n, \delta\right)
$$

where $B \in \mathbb{R}^{3 \times\left(p_{1}+p_{2}\right)}$ is an actuator configuration matrix, and $f_{c}: \mathbb{R}^{3} \times \mathbb{R}^{p_{1}} \times$ $[-\pi, \pi)^{p_{2}} \rightarrow \mathbb{R}^{p_{1}+p_{2}}$ is a function that for each velocity $v_{r}$ relates the actuator setpoints $(n, \delta)$ to a vector of forces.

As a case we consider CyberShip II which has two main propellers and two rudders aft, and one bow thruster fore; see Figure 2. The main propellers generate thrust forces $\left\{T_{1}, T_{2}\right\}$, the bow thruster generates $\left\{T_{3}\right\}$, while the rudders generate lift forces $\left\{L_{1}, L_{2}\right\}$ and drag forces $\left\{D_{1}, D_{2}\right\}$. Disregarding the drag forces, the force vector becomes

$$
f_{c}\left(v_{r}, n, \delta\right)=\left[T_{1}\left(n_{1}, u_{r}\right), T_{2}\left(n_{2}, u_{r}\right), T_{3}\left(n_{3}\right), L_{1}\left(\delta_{1}, u_{r u d, 1}\right), L_{2}\left(\delta_{2}, u_{r u d, 2}\right)\right]^{\top}
$$

where $u_{\text {rud }, i}, i=1,2$, are given below. Let the force attack points of $\left\{T_{1}, T_{2}, T_{3}\right\}$ be located at coordinates $\left\{\left(l_{x T_{1}}, l_{y T_{1}}\right),\left(l_{x T_{2}}, l_{y T_{2}}\right),\left(l_{x T_{3}}, l_{y T_{3}}\right)\right\}$ in the body-frame, and

${ }^{1}$ We will show in the next section that the rudder drag forces can be viewed as a perturbation of the hull drag in surge motion and can therefore be eliminated from the actuator model. 
likewise $\left\{\left(l_{x R_{1}}, l_{y R_{1}}\right),\left(l_{x R_{2}}, l_{y R_{2}}\right)\right\}$ for the rudders. Then the actuator configuration matrix is

$$
B=\left[\begin{array}{ccccc}
1 & 1 & 0 & 0 & 0 \\
0 & 0 & 1 & 1 & 1 \\
\left|l_{y T_{1}}\right| & -\left|l_{y T_{2}}\right| & \left|l_{x T_{3}}\right| & -\left|l_{x R_{1}}\right| & -\left|l_{x R_{2}}\right|
\end{array}\right]
$$

The propeller thrust forces $\left\{T_{1}, T_{2}\right\}$ are according to Blanke (1981) and later Fossen (1994), expressed as

$$
T_{i}=\rho d_{i}^{4} K_{T}\left(J_{i}\right)\left|n_{i}\right| n_{i}
$$

where $\rho$ is the water density, $d_{i}$ is the propeller diameter, and $K_{T}$ is a nondimensional thrust coefficient which depends on the advance ratio

$$
J_{i}=\frac{u_{a}}{n_{i}} d_{i}
$$

of thruster $i$. The ambient flow velocity $u_{a}$ is given by $u_{a}=(1-w) u_{r}$ where $w \in(0,1)$ is the wake fraction number usually assumed constant (generally it is a slowly varying dynamic variable). For a range of $J_{i}, K_{T}\left(J_{i}\right)$ is nearly linear and may be expressed according to Blanke (1981) as

$$
K_{T}\left(J_{i}\right) \approx \alpha_{0}-\alpha_{1} J_{i}=\alpha_{0}-\alpha_{1} \frac{u_{a}}{n_{i} d_{i}}
$$

where $\alpha_{0}, \alpha_{1}>0$. An approximate formula for the thrust forces is then obtained by substituting equations (23) into (22) and grouping all constants, that is

$$
T_{i}=T_{|n| n}\left|n_{i}\right| n_{i}-T_{|n| u}\left|n_{i}\right| u_{r}
$$

where $T_{|n| n}>0, T_{|n| u}>0$ are the new parameters. However, the experimental results show that the thrust force $T_{i}$ primarily is dependent on the propeller revolutions $n_{i}$ and less sensitive to the ambient flow velocity $u_{a}$. Additional accuracy is therefore obtained if equation (24) is separated into the equations

$$
T_{i}=\left\{\begin{array}{cc}
T_{|n| n}^{+}\left|n_{i}\right| n_{i}-T_{|n| u}^{+}\left|n_{i}\right| u_{r} & n_{i} \geqslant \bar{n} \\
T_{|n| n}^{-}\left|n_{i}\right| n_{i}-T_{|n| u}^{-}\left|n_{i}\right| u_{r} & n_{i} \leqslant \underline{n} \\
0 & \text { otherwise }
\end{array}\right.
$$

$i=1,2$, where

$$
\bar{n}=\max \left\{0, \frac{T_{|n| u}^{+}}{T_{|n| n}^{+}} u_{r}\right\}, \quad \underline{n}=\min \left\{0, \frac{T_{|n| u}^{-}}{T_{|n| n}^{-}} u_{r}\right\} \quad \text { and } \quad\left\{T_{|n| n}^{+}, T_{|n| u}^{+}, T_{|n| n}^{-}, T_{|n| u}^{-}\right\}
$$

are positive coefficients. For each $u_{r}$, equation (25) is a monotone function for which the inverse function becomes:

$$
n_{i}=\left\{\begin{array}{cc}
\frac{T_{|n| u}^{+}}{2 T_{|n| n}^{+}} u_{r}+\frac{1}{2 T_{|n| n}^{+}} \sqrt{\left.T_{|n| u}^{+} u_{r}\right)^{2}+4 T_{|n| n}^{+} T_{i}} & T_{i}>0 \\
0 & T_{i}=0 \\
\frac{T_{|n| u}^{-}}{2 T_{|n| n}^{\mid}} u_{r}-\frac{1}{2 T_{|n| u}^{-}} \sqrt{\left(T_{|n| u}^{-} u_{r}\right)^{2}-4 T_{|n| n}^{-} T_{i}} & T_{i}<0
\end{array}\right.
$$


The thrust force produced by the bow thruster will also depend on the velocity of the ship. However, because the exact form of this relationship is not known, we rather choose the speed independent equation used by Lindegaard \& Fossen (2003), that is,

$$
T_{3}=T_{\left|n_{3}\right| n_{3}}\left|n_{3}\right| n_{3} \quad T_{\left|n_{3}\right| n_{3}}>0
$$

This has the inverse function

$$
n_{3}=\frac{\operatorname{sgn}\left(T_{3}\right)}{T_{\left|n_{3}\right| n_{3}}} \sqrt{T_{\left|n_{3}\right| n_{3}}\left|T_{3}\right|}
$$

Finally we must find the rudder lift forces as a function of rudder angle and the relative velocity of the fluid $u_{r u d}$ at the rudder surface. From momentum theory (Lewis, 1988) it can be shown that for a positive velocity $u_{r} \geqslant 0$ then at rudder $i$, $i=1,2$,

$$
u_{r u d, i}=u_{r}+k_{u}\left(\sqrt{\frac{8}{\pi \rho d_{i}^{2}} T_{i}+u_{r}^{2}}-u_{r}\right)
$$

where $T_{i}$ is the thrust force from the preceding propeller, $d_{i}$ is the propeller diameter, and $k_{u}$ is an induced velocity factor. Normally $k_{u} \approx 0.5$ when the rudder is close to the propeller. This equation tells that for a positive surge speed and positive propeller thrust, the fluid velocity at the rudder is larger than the surge velocity $u_{r}$. However, for $T_{i}<0$ the argument inside the root may become negative. In this case we make the blanket assumption that this argument is zero. For negative surge speed we simply assume $u_{r u d, i}=u_{r}$. In summary we then have

$$
u_{r u d, i}=\left\{\begin{array}{cc}
u_{r}+k_{u}\left(\sqrt{\max \left\{0, \frac{8}{\pi \rho d_{i}^{2}} T_{i}+u_{r}^{2}\right\}}-u_{r}\right) & u_{r} \geqslant 0 \\
u_{r} & u_{r}<0
\end{array}\right.
$$

From foil theory (Newman, 1999) the lift and drag forces are modeled as

$$
\begin{aligned}
L_{i} & =\frac{\rho}{2} A_{r u d, i}^{e} C_{L}\left(\delta_{i}\right)\left|u_{r u d, i}\right| u_{r u d, i} \\
D_{i} & =-\frac{\rho}{2} A_{r u d, i}^{e} C_{D}\left(\delta_{i}\right)\left|u_{r u d, i}\right| u_{r u d, i}
\end{aligned}
$$

where $A_{\text {rud }, i}^{e}$ is the effective rudder area, $C_{L}$ is the nondimensional lift coefficient, and $C_{D}$ is the nondimensional drag coefficient. These latter coefficients are further modeled as $C_{L}\left(\delta_{i}\right)=c_{1} \delta_{i}-c_{2}\left|\delta_{i}\right| \delta_{i}$ and $C_{D}\left(\delta_{i}\right)=c_{3}\left|\delta_{i}\right|$ where $c_{1}, c_{2}$, and $c_{3}$ are positive constants. Putting this together and grouping all constants, we get the lift and drag force models

$$
\begin{aligned}
L_{i} & = \begin{cases}\left(L_{\delta}^{+} \delta_{i}-L_{|\delta| \delta}^{+}\left|\delta_{i}\right| \delta_{i}\right)\left|u_{\text {rud }, i}\right| u_{\text {rud }, i} & u_{\text {rud }, i} \geqslant 0 \\
\left(L_{\delta}^{-} \delta_{i}-L_{|\delta| \delta}^{-}\left|\delta_{i}\right| \delta_{i}\right)\left|u_{\text {rud }, i}\right| u_{\text {rud }, i} & u_{\text {rud }, i}<0\end{cases} \\
D_{i} & =-D_{|\delta|}\left|\delta_{i}\right|\left|u_{\text {rud }, i}\right| u_{\text {rud }, i}
\end{aligned}
$$

where $\left\{L_{\delta}^{+}, L_{|\delta| \delta}^{+}, L_{\delta}^{-}, L_{|\delta| \delta}^{-}, D_{|\delta|}\right\}$ are positive coefficients. We allow the lift forces to have different coefficients for positive and negative velocities. The drag forces should now be added to the propeller thrust forces, $T_{i}+D_{i}$, in the overall actuator 


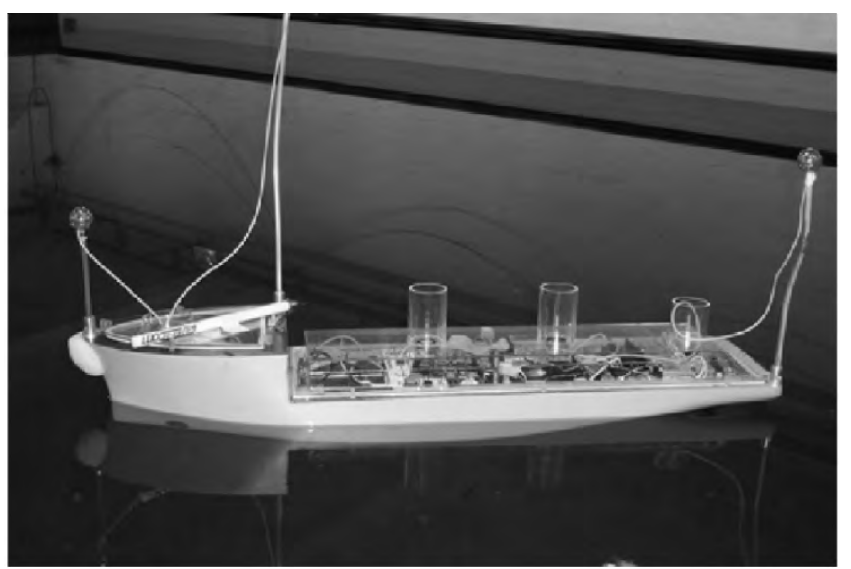

Figure 3. A picture of CyberShip II in the Marine Cybernetics Laboratory at NTNU.

model. However, since $D_{i}$ depends on $T_{i}$ through $u_{\text {rud, } i}$ this expression becomes excessively complicated. To make it less complicated, experimental data suggest that $D_{i}$ can be viewed as a perturbation of the hull drag force $d_{1}\left(v_{r}\right)$ in surge. Assuming that a robust manoeuvering controller is able to deal with this perturbation, we do not consider it hereafter. The inverse function of equation (32) is

$$
\delta_{i}=\left\{\begin{array}{cc}
\frac{\operatorname{sgn}\left(L_{i}\right)}{2 L_{|\delta| \delta}^{+}}\left(L_{\delta}^{+}-\frac{1}{u_{r u d, i}^{2}} \sqrt{\left(L_{\delta}^{+} u_{r u d, i}^{2}\right)^{2}-4 L_{|\delta| \delta}^{+} u_{r u d, i}^{2}\left|L_{i}\right|}\right) & u_{r u d, i} \geqslant \varepsilon \\
0 & \left|u_{r u d, i}\right|<\varepsilon \\
\frac{-\operatorname{sgn}\left(L_{i}\right)}{2 L_{|\delta| \delta}^{-}}\left(L_{\delta}^{-}-\frac{1}{u_{r u d, i}^{2}} \sqrt{\left(L_{\delta}^{-} u_{r u d, i}^{2}\right)^{2}-4 L_{|\delta| \delta}^{-} u_{r u d, i}^{2}\left|L_{i}\right|}\right) & u_{r u d, i} \leqslant-\varepsilon
\end{array}\right.
$$

where we have introduced an $\varepsilon$-neighbourhood around the non-effective point $u_{r u d, i}=0$ to avoid division by zero.

\section{System Identification}

The Marine Cybernetics Laboratory (MCLab) is an experimental facility ${ }^{2}$ for testing of ships, rigs, underwater vehicles, and propulsion systems at the Centre for Ships and Ocean Structures (CESOS) at the Norwegian University of Science and Technology (NTNU). The dimensions of the basin are $\mathrm{L} \times \mathrm{B} \times \mathrm{D}=40 \mathrm{~m} \times 6.45 \mathrm{~m} \times$ $1.5 \mathrm{~m}$, and it is equipped with a towing carriage, a position measurement system, and a wave maker system, while a wind and current system are under construction.

CyberShip II (CS2; see Figure 3 is a 1:70 scale replica of a supply ship for the North Sea. Its mass is $m=23.8 \mathrm{~kg}$, its length is $L_{C S 2}=1.255 \mathrm{~m}$, and its breadth is $B_{C S 2}=0.29 \mathrm{~m}$. It is fully actuated with two main propellers and two rudders aft, and one bow thruster; see Figure 2. It is further equipped with a PC104-bus driven by a $\mathrm{QNX}^{\circledR}$ real-time operating system which controls the internal hardware achitecture and communicates with onshore computers through a WLAN. For position and

\footnotetext{
${ }^{2}$ The MCLab is a Marie Curie EU training site.
} 
attitude measurements, four cameras onshore in the MCLab observe three infrared emitters on the ship, and a kinematic computer algorithm calculates the 6 degreesof-freedom (6 DOF) data. The accuracy of these measurements are very high which means that the corresponding velocities can be estimated with high precision to render a full state feedback design possible. To facilitate real-time feedback control of the ship, Opal RT-Lab ${ }^{\circledR}$ is used for rapid prototyping of a desired control structure programmed in Matlab ${ }^{\circledR}$ and Simulink ${ }^{\circledR}$. For execution of free-running experiments, a LabVIEW ${ }^{\circledR}$ interface has been developed for commanding and monitoring the ship.

Since we do not expose the ship to any currents or exogenous disturbances in the model basin, the CS2 ship model becomes

$$
M \dot{v}+C(v) v+D(v) v=B f_{c}(v, n, \delta)
$$

where the parameters in $M_{R B}, M_{A}, D_{L}, D_{N L}(v), B$ and $f_{c}(v, n, \delta)$ must be identified. We choose the following strategy:

(1) The matrices $M_{R B}, M_{A}$, and $B$ are found from the main particulars of CS2 (weight, mass distribution, lengths, area, volume, etc.)

(2) By towing CS2 at different constant surge and sway velocities, with $f_{c}=0$, and measuring the average towing forces, one can use least square interpolation to find the damping parameters in $D_{L}$ and $D_{N L}(v)$ that are excited by pure surge and sway motions; see Figure 4.

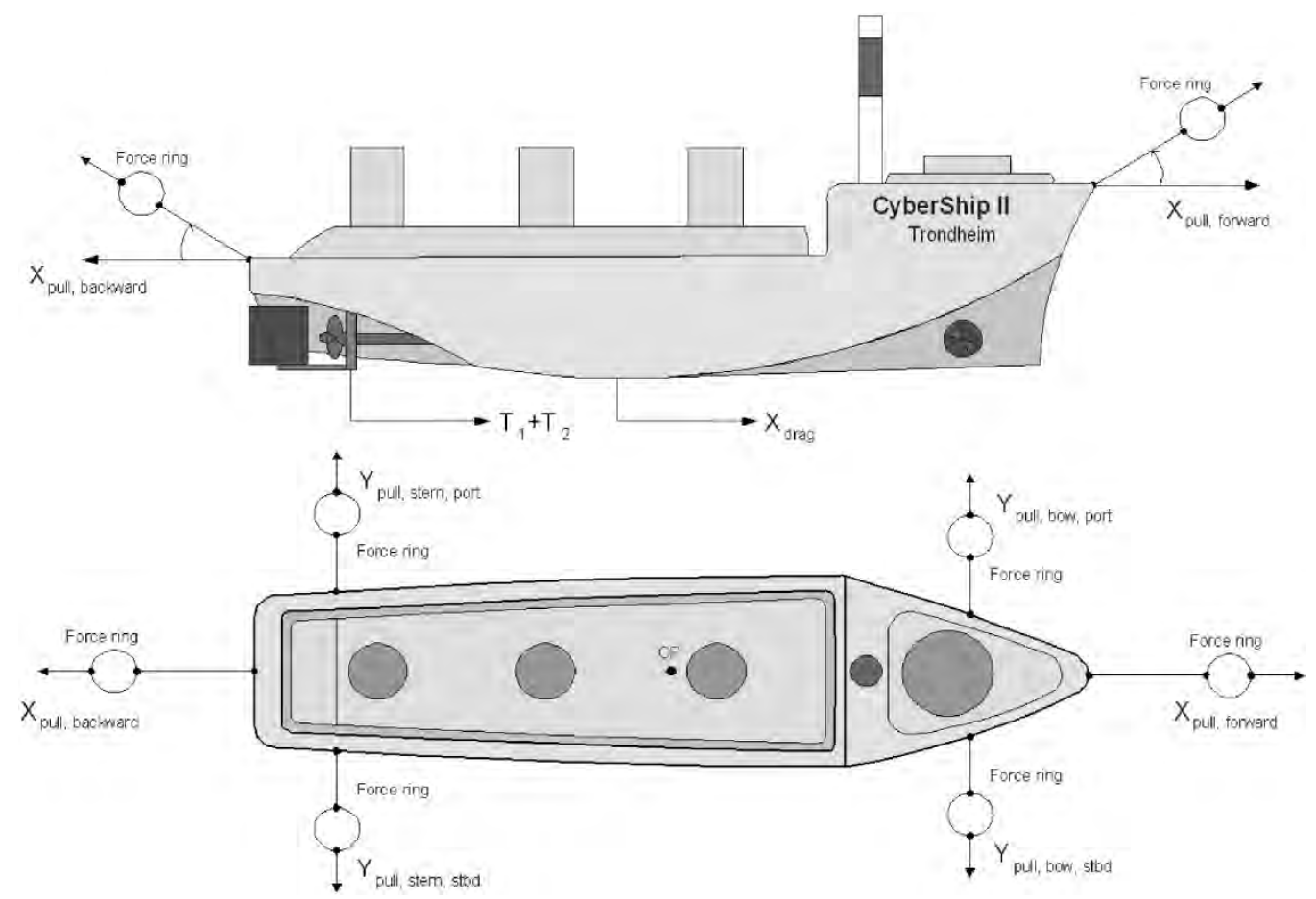

Figure 4. Two force rings, forward and backward, were applied to measure the drag and propulsion forces when towing CyberShip II longitudinally at different speeds. Four force rings, two port and two starboard, were used to measure the drag force and moment for lateral motion. 
Table 1. Mass-related parameters with respect to CP for CyberShip II

\begin{tabular}{lrrrrr}
\hline$m$ & 23.800 & $Y_{\dot{v}}$ & -10.0 & $\left(l_{x T_{1}}, l_{y_{1}}\right)$ & $(-0.499,-0.078)$ \\
$I_{z}$ & 1.760 & $Y_{\dot{r}}$ & -0.0 & $\left(l_{x T_{2}}, l_{y T_{2}}\right)$ & $(-0.499,0.078)$ \\
$x_{g}$ & 0.046 & $N_{\dot{v}}$ & -0.0 & $\left(l_{x T_{3}}, l_{y T_{3}}\right)$ & $(0.466,0.000)$ \\
$X_{\dot{u}}$ & -2.000 & $N_{\dot{r}}$ & -1.0 & $\left(l_{x R_{1}}, l_{y R_{1}}\right)$ & $(-0.549,-0.078)$ \\
& & & & $\left(l_{x R_{2}}, l_{y R_{2}}\right)$ & $(-0.549,0.078)$ \\
\hline
\end{tabular}

(3) When the damping parameters for pure surge and sway motions are known, the actuator parameters in $f_{c}(v, n, \delta)$ are found by repeating the above towing experiments at different thruster revolutions and rudder angles.

(4) The remaining parameters are those damping coefficients excited by the yaw rate. Lacking equipment for turning experiments and moment measurements on the towing carriage, we choose to use adaptive estimation in free-running adaptive manoeuvering experiments to find those remaining parameters.

The parameters in the rigid-body system inertia matrix $M_{R B}$ and the input matrix $B$ are found from straight-forward measurements of the main particulars of the ship, that is, its dimensions, weight, mass distribution, volume, area, and the actuator setup. The zero frequency added mass coefficients in $M_{A}$ can be found from semiempirical formulas or simple engineering 'rules-of-thumb'. For commercial ships, however, strip theory is usually applied (Faltinsen, 1990). This requires a ship geometry computation program that produces a geometry file which is fed into a hydrodynamic computation program based on strip theory. Nevertheless, for CS2 these parameters have all been roughly estimated beforehand by Lindegaard (2003), and their values are given in Table 1. The ship model used by Lindegaard (2003) was for DP using a linear damping model according to equation (17). Since we seek a nonlinear representation of the damping effects, the DP values cannot be used. The system identification procedure next will therefore be concerned with the damping and actuator coefficients.

The parameters to be identified in the surge direction are $\left\{X_{u}, X_{|u| u}, X_{\text {uuu }}\right\}$ and $\left\{T_{|n| n}^{+}, T_{|n| u}^{+}, T_{|n| n}^{-}, T_{|n| u}^{-}\right.$. Using the towing carriage, CS2 was pulled both forward and backward at different constant speeds, and for each run the average pull force $X_{p u l l}$ was measured and recorded; see Figure 4. Since $\dot{u}=v=r=0$, and letting $n_{1}=n_{2}$ at each run, we have for pure surge motion that

$$
0= \begin{cases}X_{\text {pull }}+X_{\text {drag }}+2 T_{|n| n}^{-}\left|n_{1}\right| n_{1}-2 T_{|n| u}^{-}\left|n_{1}\right| u & n_{1}<0 \\ X_{\text {pull }}+X_{\text {drag }}+2 T_{|n| n}^{+}\left|n_{1}\right| n_{1}-2 T_{|n| u}^{+}\left|n_{1}\right| u & n_{1} \geqslant 0\end{cases}
$$

where $X_{\text {drag }}=X_{u} u+X_{|u| u}|u| u+X_{u u u} u^{3}$. Setting this up as a linear set of equations, $A x=b$, where $x$ contains the unknown parameters, $A$ contains the applied speeds $u$ and propeller $\operatorname{rps} n_{1}$, and $b$ contains the corresponding measured forces $X_{\text {pull }}$, the unknown coefficients are calculated by a least square fit. For $n_{1}=n_{2}=0$ then $X_{\text {drag }}=-X_{\text {pull }}$. Figure 5 shows these measured forces and the corresponding interpolation. In addition it shows the linear DP curve $X_{\text {drag }}=X_{u} u$ fitted to those measured points that are within the slow speed region $u \in[-0.15,0.15]$. Clearly, there is a large discrepancy for higher speeds. Having the nominal drag forces for $n_{1}=n_{2}=0$, then the same towing experiments are repeated for different propeller revolutions. These were chosen as $n_{1}=n_{2} \in\{ \pm 200, \pm 500, \pm 1000, \pm 2000\}$, and the thrust forces were estimated from $2 T_{1}=-X_{\text {pull }}-X_{\text {drag }}$. The result is shown in Figure 6 where it is 


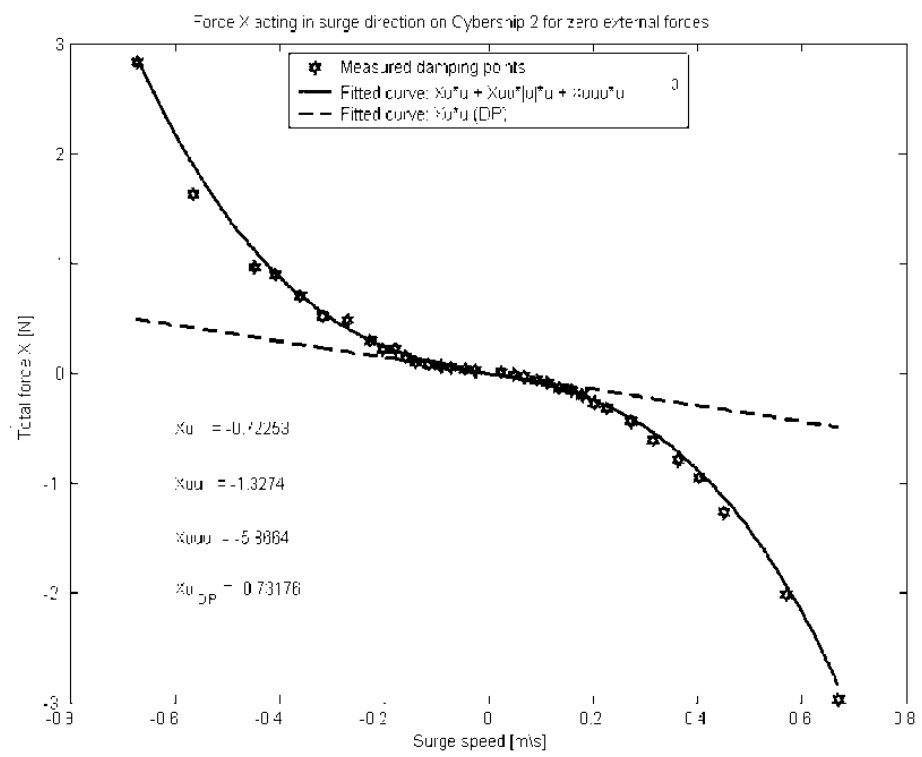

Figure 5. Measured drag forces of CyberShip II for $n_{1}=n_{2}=0$ at different speeds and the corresponding fitted nonlinear curve as well as a linear curve for DP.

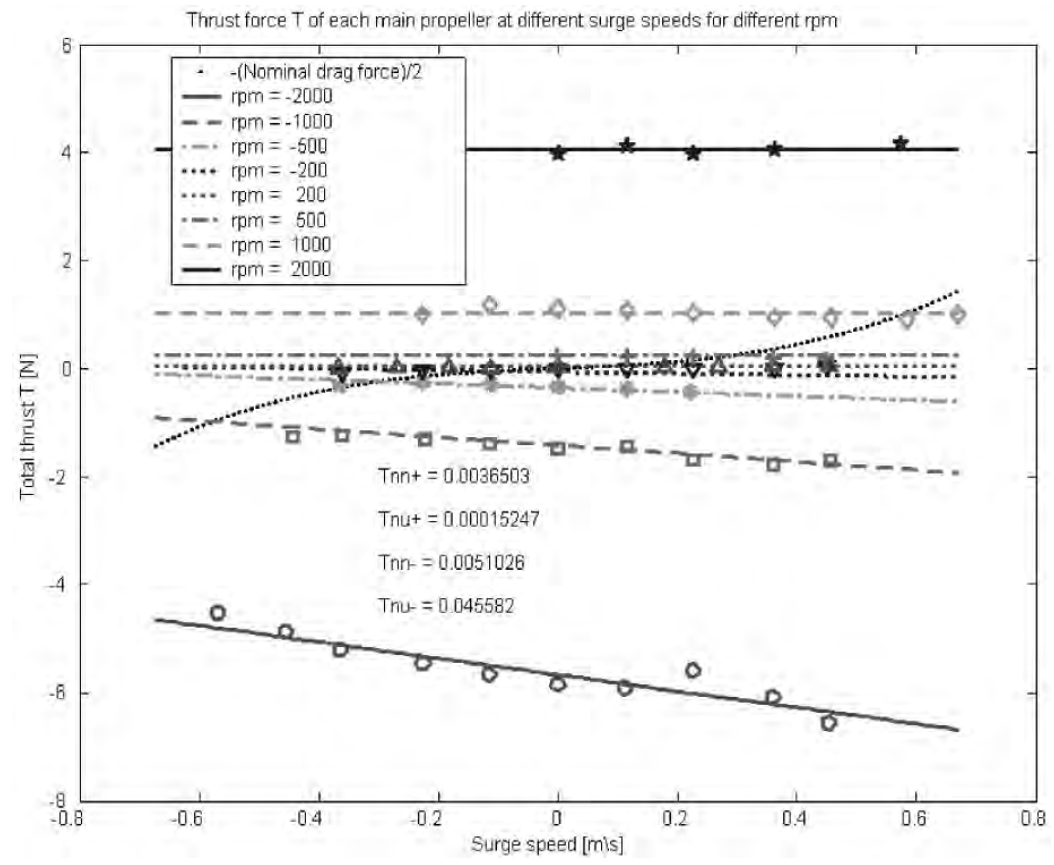

Figure 6. Measured and interpolated thrust forces $T_{1}=T_{2}$ for different propeller revolutions at different speeds for CyberShip II. 


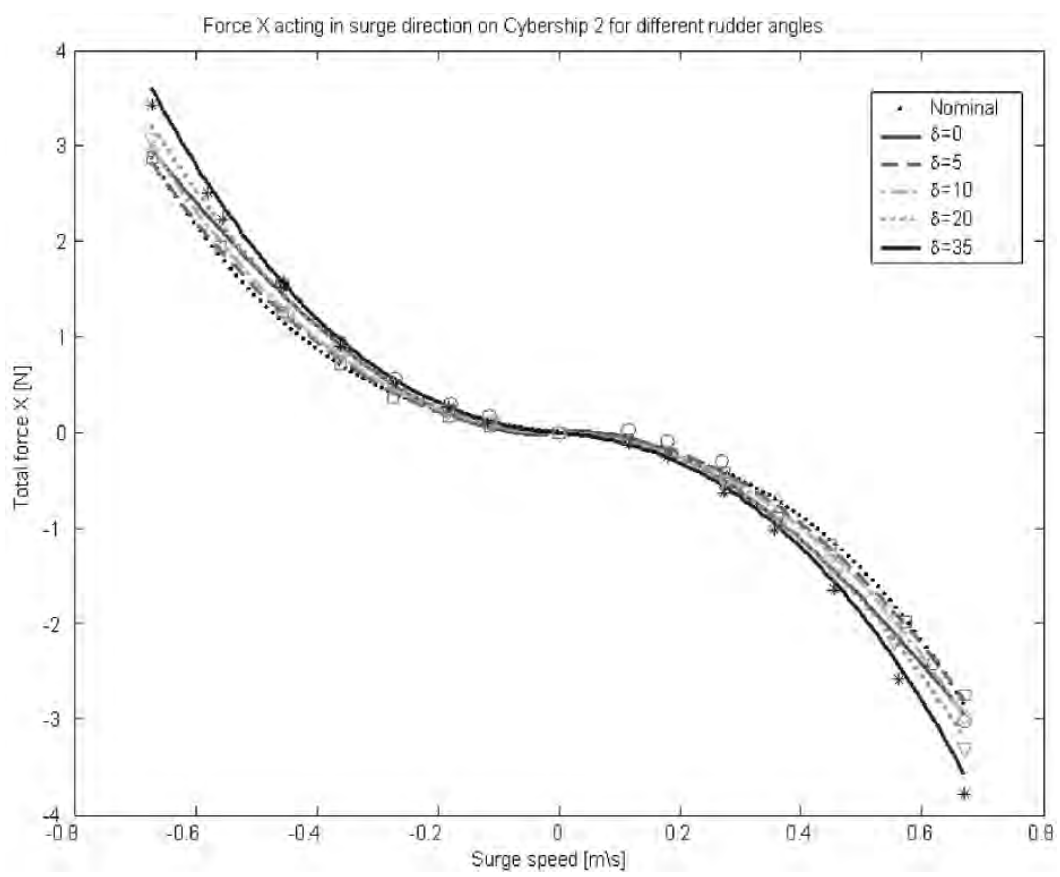

Figure 7. The measured and curve fitted drag forces for different rudder angles $\delta_{1}=\delta_{2}$ for CyberShip II.

observed that for each revolution set-point, the surge speed has very little effect at positive revolutions, while for negative revolutions the slope is higher. Figure 7 shows how the rudders affect the drag in surge motion at different speeds. This justifies the argument, previously discussed, of not including the the rudder drag force $D_{i}$ in the actuator model, but rather viewing it as perturbations of the nominal drag coefficients. A robust control design should compensate for this.

The next step is to identify the parameters $\left\{Y_{v}, Y_{|v| v}, N_{v}, N_{|v| v}\right\}$ which can be found from pure sway motion measurements. In this case we have $\dot{v}=u=r=0$, and the force equation becomes $Y_{\text {pull }}+Y_{\text {draq }}=0$ where $Y_{\text {drag }}=Y_{v} v+Y_{|v| v}|v| v$. Force rings are set up according to Figure 4 to measure the pull forces at both positive and negative sway speeds. The full set of measurements constitutes a set of linear equations that are solved by least square minimization, see Figure 8 . These measurements are also used to identify the moment coefficients $\left\{N_{v}, N_{|v| v}\right\}$. The moment equation is $N_{\text {pull }}+N_{\text {drag }}=0$ where $N_{\text {drag }}=N_{v} v+N_{|v| v}|v| v$. Let the moment arms from CP to the stern and bow measurement points for $Y_{\text {pull }}$ be $l_{\text {stern }}$ and $l_{\text {bow }}$ respectively; see Figure 4. Then $v>0 \Rightarrow N_{\text {pull }}=Y_{\text {pull, bow, stbd }} \cdot l_{\text {bow }}-Y_{\text {pull, stern, stbd }} \cdot l_{\text {stern }}$ and $v<0 \Rightarrow$ $N_{\text {pull }}=Y_{\text {pull, stern, port }} \cdot l_{\text {stern }}-Y_{\text {pull, bow, port }} \cdot l_{\text {bow }}$. The result of this interpolation is shown in Figure 9.

To identify the rudder lift forces $\left\{L_{\delta}^{+}, L_{|\delta| \delta}^{+}, L_{\delta}^{-}, L_{|\delta| \delta}^{-}\right\}$, CS2 is towed forward with $\delta_{1}=\delta_{2}<0$ and backward with $\delta_{1}=\delta_{2}>0$ for different (equal) rudder angles, and for each run the average force $Y_{\text {pull, stern, stbd }}$ is recorded. The moment equation is $N_{\text {pull }}+N_{\text {lift }}=0$ where $N_{\text {lift }}=2\left|l_{x R_{1}}\right| L_{1}$ and $L_{1}$ is given by equation (32) with $u_{r u d}=u$. For these runs, the sideslip angle $\beta=\arctan (v / u) \approx 0$ such that we can assume that $N_{\text {pull }} \approx-Y_{\text {pull,stern, stbd }} \cdot l_{\text {stern }}$, that is, not affected by the moment arms from $X_{\text {pull }}$. 


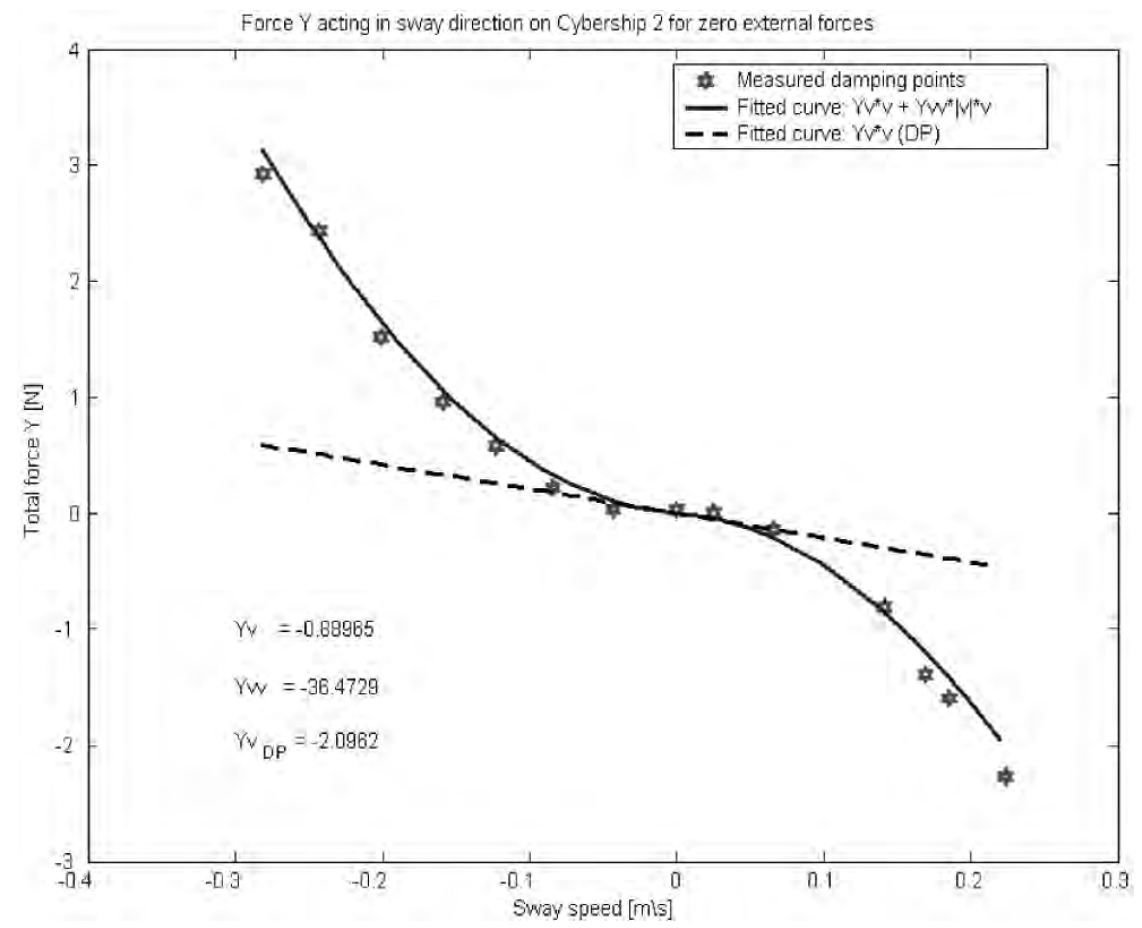

Figure 8. Measured drag forces in sway motion and the corresponding fitted nonlinear curve as well as a linear curve for DP for CyberShip II.

Separating positive and negative motion according to equation (32), Figure 10 shows that the rudders are most effective in forward motion. Finally, we repeat the same experiment for the bow thruster to find the parameter $\left\{T_{\left|n_{3}\right| n_{3}}\right\}$ in equation (27). Unfortunately, the sideslip angle $\beta$ was rather high in these runs so that hull drag distorted the measurements for higher speeds. Nevertheless, $Y_{\text {pull, bow, stbd }}$ was measured, and since equation (27) is an odd function it is enough to test with negative revolutions for $n_{3}$. Figure 11 shows the measured points and the weighted least square fitted curve.

To sum up, the parameters identified thus far are given in Tables 1 and 2.

Since no yaw motion was induced in these towing experiments, the parameters $\left\{Y_{r}, Y_{|r| v}, Y_{|v| r}, Y_{|r| r}, N_{r}, N_{|r| v}, N_{|v| r}, N_{|r| r}\right\}$ are yet to be identified. We leave these to be estimated in the adaptive manoeuvering controller developed and experimentally tested in the next section.

\section{Adaptive Ship Manoeuvering With Experiments}

We consider the dynamic ship model equations (1) and (35) which for $\tau=B f_{c}(v, n, \delta)$ can be rewritten as

$$
\begin{aligned}
\dot{\eta} & =R(\psi) v \\
M \dot{v} & =\tau-C(v) v+g(v)+\Phi(v) \varphi
\end{aligned}
$$




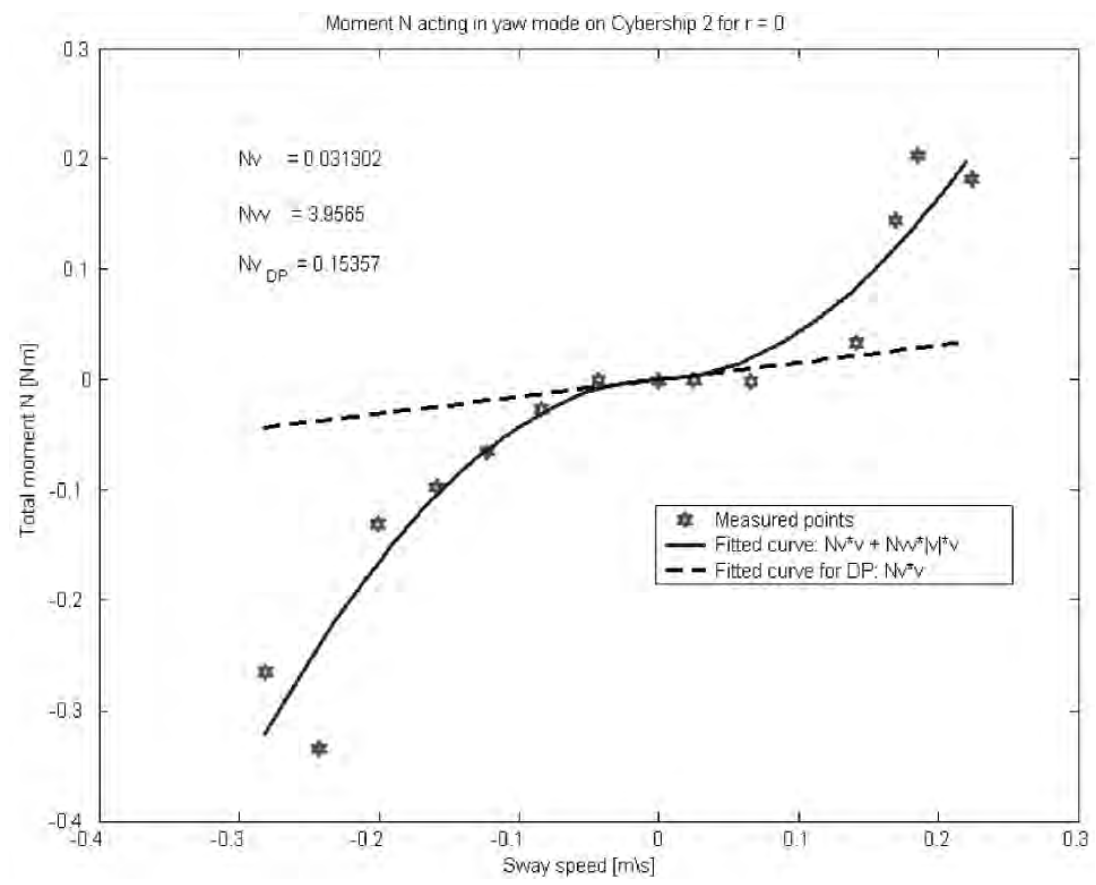

Figure 9. Measured drag moments in sway motion and the corresponding fitted nonlinear curve as well as a linear curve for DP for CyberShip II.

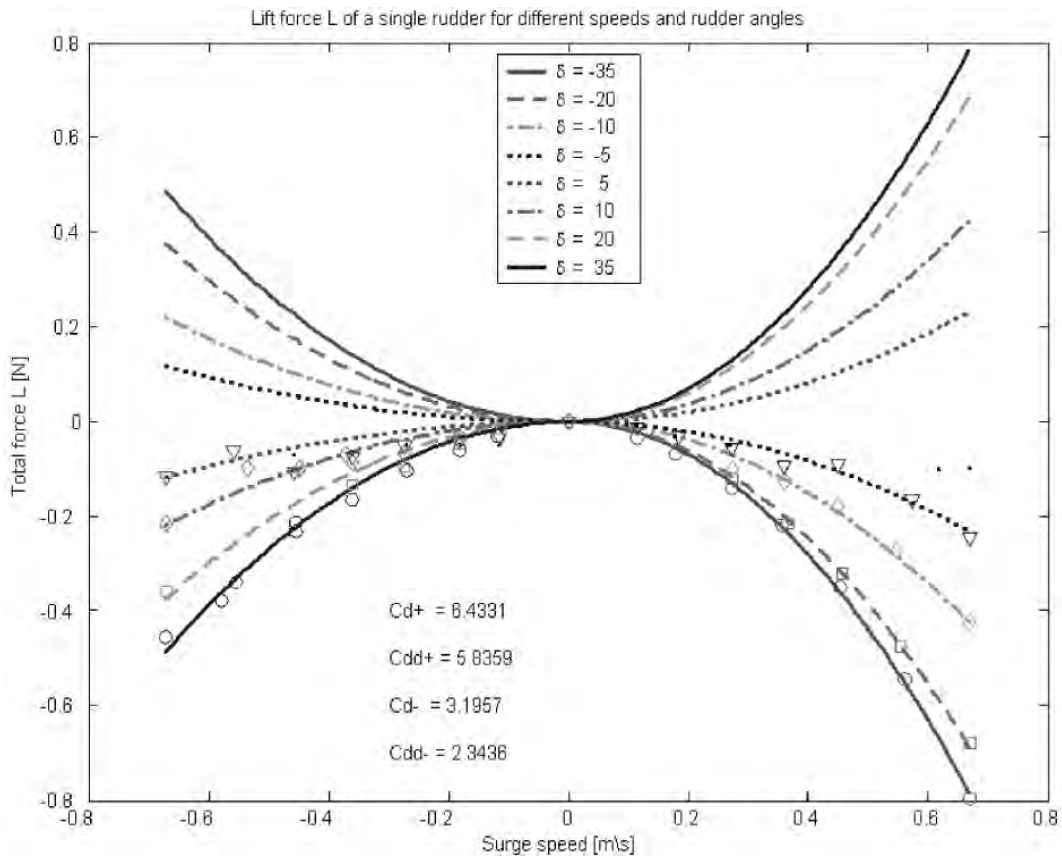

Figure 10. Curve interpolation to the measured lift forces for the rudders. Notice that CyberShip II generate more lift force in forward motion than backward motion. 


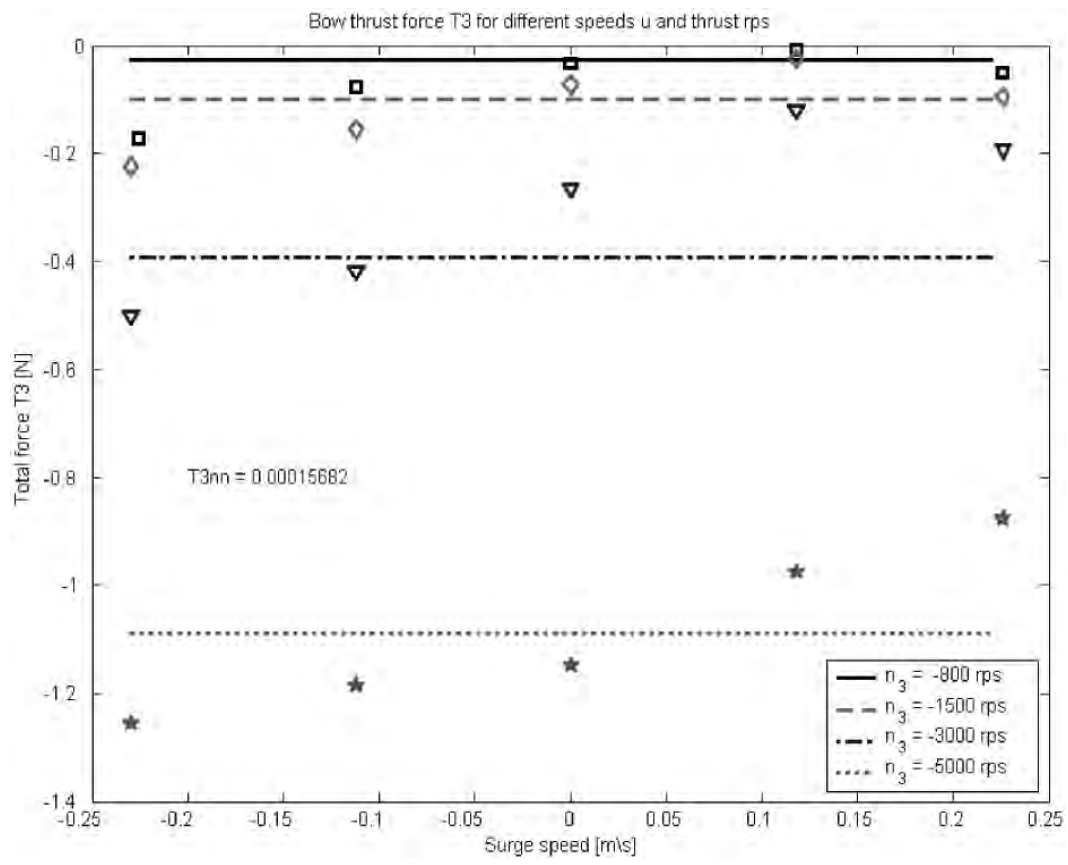

Figure 11. Measured and interpolated bow thrust force $T_{3}$ for forward and backward motion of CyberShip II and different negative propeller revolutions.

Table 2. Experimentally identified parameters for CyberShip II

\begin{tabular}{llllll}
\hline$X_{u}$ & -0.72253 & $N_{v}$ & 0.03130 & $T_{|n| n}^{+}$ & $3.65034 \mathrm{E}-3$ \\
$X_{|u| u}$ & -1.32742 & $N_{|v| v}$ & 3.95645 & $T_{|n| u}^{+}$ & $1.52468 \mathrm{E}-4$ \\
$X_{u u u}$ & -5.86643 & $L_{\delta}^{+}$ & 6.43306 & $T_{|n| n}^{-}$ & $5.10256 \mathrm{E}-3$ \\
$Y_{v}$ & -0.88965 & $L_{|\delta| \delta}^{+}$ & 5.83594 & $T_{|n| u}^{-}$ & $4.55822 \mathrm{E}-2$ \\
$Y_{|v| v}$ & -36.47287 & $L_{\delta}^{-\mid}$ & 3.19573 & $T_{\left|n_{3}\right| n_{3}}$ & $1.56822 \mathrm{E}-4$ \\
& & $L_{|\delta| \delta}^{-}$ & 2.34356 & & \\
\hline
\end{tabular}

where $g(v)$ is the known part of $-D(v) v$ and

$$
\begin{aligned}
\varphi & :=\left[Y_{|r| v}, Y_{r}, Y_{|v| r}, Y_{|r| r}, N_{|r| v}, N_{r}, N_{|v| r}, N_{|r| r}\right]^{\top} \\
\Phi(v):= & {\left[\begin{array}{cccccccc}
0 & 0 & 0 & 0 & 0 & 0 & 0 & 0 \\
|r| v & r & |v| r & |r| r & 0 & 0 & 0 & 0 \\
0 & 0 & 0 & 0 & |r| v & r & |v| r & |r| r
\end{array}\right] }
\end{aligned}
$$

are the vector of unknown parameters and the regressor matrix, respectively, so that $g(v)+\Phi(v) \varphi=-D(v) v$. The objective is to design a robust adaptive control law that ensures tracking of $\eta(t)$ to a time-varying reference $\eta_{d}(t)$ while adapting the parameters $\varphi$. 


\section{Remark 1}

Using adaptive tracking to estimate unknown parameters will not in general guarantee convergence to the true values. This is only obtained if the inputs to the closed-loop system (references and disturbances) are persistently exciting the regressor matrix (Anderson et al., 1986). Consequently, this step in the parameter identification strategy involves most uncertainty. Nonetheless, the success in the design of a robust tracking control law with subsequent accurate tracking in experiments indicate that 100\% parameter accuracy of the model is not necessary. Using the obtained (numerical) model with integral action to compensate for the bias $\mathrm{b}$ in equation (15) for control design should guarantee success in practical implementations.

The time-varying reference $\eta_{d}(t)$ must trace out a desired path on the surface as well as satisfying a desired speed specification along the path. Such problems are conveniently solved according to the methodology in Skjetne et al. $(2004 a, b)$, where the tracking objective is divided into two tasks. Instead of constructing a desired reference $\eta_{d}(t)$ that contains both the path and speed objectives in one package, one can keep these objectives separate by solving the manoeuvering problem.

Using $\theta$ as a scalar parametrization variable, we want the desired path to be an ellipsoid with heading along the tangent vector, that is,

$$
\eta_{d}(\theta)=\left[\begin{array}{lll}
x_{d}(\theta), & y_{d}(\theta), & \arctan \left(\frac{y_{d}^{\theta}(\theta)}{x_{d}^{\theta}(\theta)}\right)
\end{array}\right]^{\top}
$$

where $x_{d}(\theta)=5+4.5 \cos ((\pi / 180) \theta)$ and $y_{d}(\theta)=-0.75-2.25 \sin ((\pi / 180) \theta)$. For the speed specification, we want the surge speed $u(t)$ to track a desired surge speed $u_{d}(t)$ which is adjustable online by an operator. This latter objective can be translated into a speed assignment for $\dot{\theta}(t)$ by noting the relationship

$$
u_{d}(t)=\sqrt{x_{d}^{\theta}(\theta(t))^{2}+y_{d}^{\theta}(\theta(t))^{2}} \dot{\theta}(t)
$$

The corresponding speed assignment for $\dot{\theta}$ becomes

$$
v_{s}(\theta, t):=\frac{u_{d}(t)}{\sqrt{x_{d}^{\theta}(\theta)^{2}+y_{d}^{\theta}(\theta)^{2}}}
$$

which has the partial derivatives

$$
\begin{aligned}
& v_{s}^{\theta}(\theta, t)=\frac{-\left[x_{d}^{\theta}(\theta) x_{d}^{\theta^{2}}(\theta)+y_{d}^{\theta}(\theta) y_{d}^{\theta^{2}}(\theta)\right]}{\left[x_{d}^{\theta}(\theta)^{2}+y_{d}^{\theta}(\theta)^{2}\right]^{3 / 2}} u_{d}(t) \\
& v_{s}^{t}(\theta, t)=\frac{\dot{u}_{d}(t)}{\sqrt{x_{d}^{\theta}(\theta)^{2}+y_{d}^{\theta}(\theta)^{2}}}
\end{aligned}
$$

where $u_{d}(t)$ and $\dot{u}_{d}(t)$ are provided online by the operator, for example by filtering a constant reference $u_{R E F}$ through a reference filter.

The control objective is then, according to Skjetne et al.(2004a,b), formally stated as a manoeuvering problem:

1. Geometric Task: Force the ship position and heading $\eta$ to converge to and follow the desired path $\eta_{d}(\theta)$,

$$
\lim _{t \rightarrow \infty}\left|\eta(t)-\eta_{d}(\theta(t))\right|=0
$$


2. Dynamic Task: Force the path speed $\dot{\theta}$ to converge to the desired speed assignment $v_{s}(\theta, t)$,

$$
\lim _{t \rightarrow \infty}\left|\dot{\theta}(t)-v_{s}(\theta(t), t)\right|=0
$$

Note that the dynamic task can be solved identically by letting $\dot{\theta}=v_{s}(\theta, t)$ be a dynamic state in the control law, called a tracking update law, which is decoupled from the rest of the dynamics of the ship. Other update laws are also possible based on the results in Skjetne et al. (2004a).

The manoeuvering control design is based on adaptive backstepping (Krstic et al., 1995). A complete adaptive design procedure with stability analysis for solving the manoeuvering problem is reported in Skjetne et al. (2004a) where CS2 is used in a case study. This gives the internal control signals

$$
\begin{aligned}
z_{1} & :=R(\psi)^{\top}\left(\eta-\eta_{d}(\theta)\right) \\
z_{2} & :=v-\alpha_{1}(\eta, \theta, t) \\
\alpha_{1} & =-K_{p} z_{1}+R(\psi)^{\top} \eta_{d}^{\theta}(\theta) v_{s}(\theta, t) \\
\sigma_{1} & =-K_{p}\left(\dot{R}(r)^{\top} R(\psi) z_{1}+v\right)+\dot{R}(r)^{\top} \eta_{d}^{\theta}(\theta) v_{s}(\theta, t)+R(\psi)^{\top} \eta_{d}^{\theta}(\theta) v_{s}^{t}(\theta, t) \\
\alpha_{1}^{\theta} & =-K_{p} R(\psi)^{\top} \eta_{d}^{\theta}(\theta)+R(\psi)^{\top}\left[\eta_{d}^{\theta^{2}}(\theta) v_{s}(\theta, t)+\eta_{d}^{\theta}(\theta) v_{s}^{\theta}(\theta, t)\right]
\end{aligned}
$$

where the error vector $z_{1}$ is rotated to the body-frame for convenience. This means that the controller gains are not dependent on the ship heading (which is more intuitive since a control technician will himself be located in the body-frame when tuning the gains). The control law, the adaptive update law, and the manoeuvering update law are given in Table 3 , where $\hat{\varphi}$ is the parameter estimate, $K_{p}=K_{p}^{\top}>0$, $K_{d}=K_{d}^{\top}>0$, and $\Gamma=\Gamma^{\top}>0$ are controller gain matrices.

Finding the optimal actuator set-points $(n, \delta)$ for each commanded input $\tau$ in equation (20) is termed control allocation. The simplest approach is to solve an unconstrained least-square optimization problem by using the generalized pseudoinverse and the inverse functions (26), (28) and (34), that is,

$$
(n, \delta)=f_{c}^{-1}(v, B \dagger \tau)
$$

Table 3. Manoeuvering control and guidance system for CyberShip II

$$
\begin{aligned}
& \text { Control: } \\
& \dot{\hat{\varphi}}=\Gamma \Phi(v)^{\mathrm{T}} z_{2} \\
& \dot{\theta}=v_{s}(\theta, t) \\
& \tau=-z_{1}-K_{d} z_{2}-g(v)-\Phi(v) \hat{\varphi}+C(v) \alpha_{1}+M \sigma_{1}+M \alpha_{1}^{\theta} v_{s}(\theta, t) \\
& \text { input }=\left\{\begin{array}{c}
(\eta, v),\left(\eta_{d}(\theta), \eta_{d}^{\theta}(\theta), \eta_{d}^{\theta^{2}}(\theta)\right), \\
\left(v_{s}(\theta, t), v_{s}^{\theta}(\theta, t), v_{s}^{t}(\theta, t)\right)
\end{array}\right\} \\
& \text { output }=\{\tau, \theta\} \\
& \text { Guidance: } \\
& \text { input }=\left\{\theta, u_{d}(t), \dot{u}_{d}(t)\right\} \\
& \text { output }=\left\{\begin{array}{c}
\left(\eta_{d}(\theta), \eta_{d}^{\theta}(\theta), \eta_{d}^{\delta^{2}}(\theta)\right), \\
\left(v_{s}(\theta, t), v_{s}^{\theta}(\theta, t), v_{s}^{t}(\theta, t)\right)
\end{array}\right\}
\end{aligned}
$$




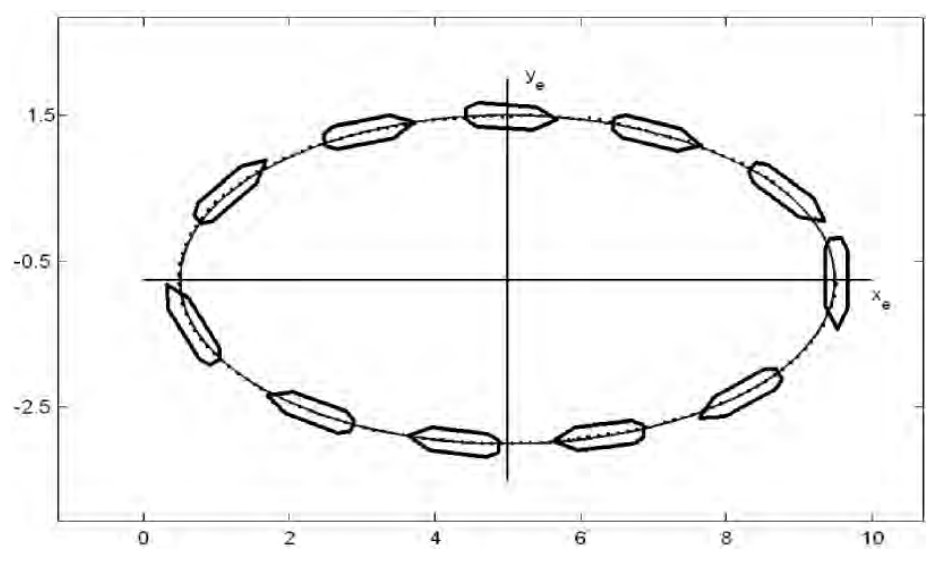

Figure 12. CyberShip II tracing the desired path.

where $B_{\dagger}^{\dagger}=W^{-1} B^{\top}\left(B W^{-1} B^{\top}\right)^{-1}$ is the generalized pseudo-inverse with a weight matrix $W$ (Fossen, 2002, Chapter 7.5). Experience has shown, though, that using the pseudo-inverse does not result in good control allocation. A more advanced method is to use constrained optimization techniques. For CS2 this has been developed and reported by Lindegaard \& Fossen (2003), Johnsen et al. (2003), where the routine developed by the former authors has been used in these experiments.

For the experiment, the controller settings were $K_{p}=\operatorname{diag}(0.5,2.0,1.5)$, $K_{d}=\operatorname{diag}(8,25,18)$, and $\Gamma=\operatorname{diag}(8,4,8,8,8,4,8,8)$. The initial condition for the parameter update was $\hat{\varphi}(0)=0$. The ship was first put to rest in dynamic positioning (zero speed) at $\eta_{d}(0)$, and then the ship was commanded online to move along the path with $u_{R E F}=0.15 \mathrm{~m} / \mathrm{s}$ for 22 rounds before we commanded it to come to a stop again. The experiment was conducted on calm water without environmental disturbances (sea state code 0) since we use and wish to estimate zero frequency hydrodynamic parameters.

Figure 12 shows how CS2 accurately traced the path (in the time interval $t \in[808,950] \mathrm{s})$. In the experiment we experienced problems with position measurement outages along the upper side of the path. This accounts for the transients at $\mathrm{t} \approx 500 \mathrm{~s}$ in the surge speed response seen in Figure 13. The way the manoeuvering problem is posed, accurate path following has priority over accurate speed tracking. Nevertheless, it is seen in Figure 13 that CS2 tracks the commanded speed quite well. Figure 14 shows the adaptive parameter estimates of $\hat{\varphi}(t)$. We observe a rapid change and a subsequent slow convergence to new values. We believe those values are close to the true values for the nominal surge speed $u \approx 0.15 \mathrm{~m} / \mathrm{s}$ and moving along this ellipsoidal path. It is likely that the parameter convergence will be different for different paths and speeds. Nonetheless, we adopt these values as approximate values for the remaining parameters in the manoeuvering model for CS2; see Table 4.

This robust adaptive manoeuvering design with experiments also illustrates that $100 \%$ numerically correct values for the hydrodynamic parameters are not necessary to achieve accurate tracing of the path. Table 5 shows the standard deviations of the error signals in $z_{1}$. The most important variable for path keeping is $z_{12}$ since this is an approximate measure of the cross-track error (provided the ship is pointed along the path, $z_{13} \approx 0$ ). An accuracy of $2.26 \mathrm{~cm}$ is $7.8 \%$ of the ship breadth and acceptable. 


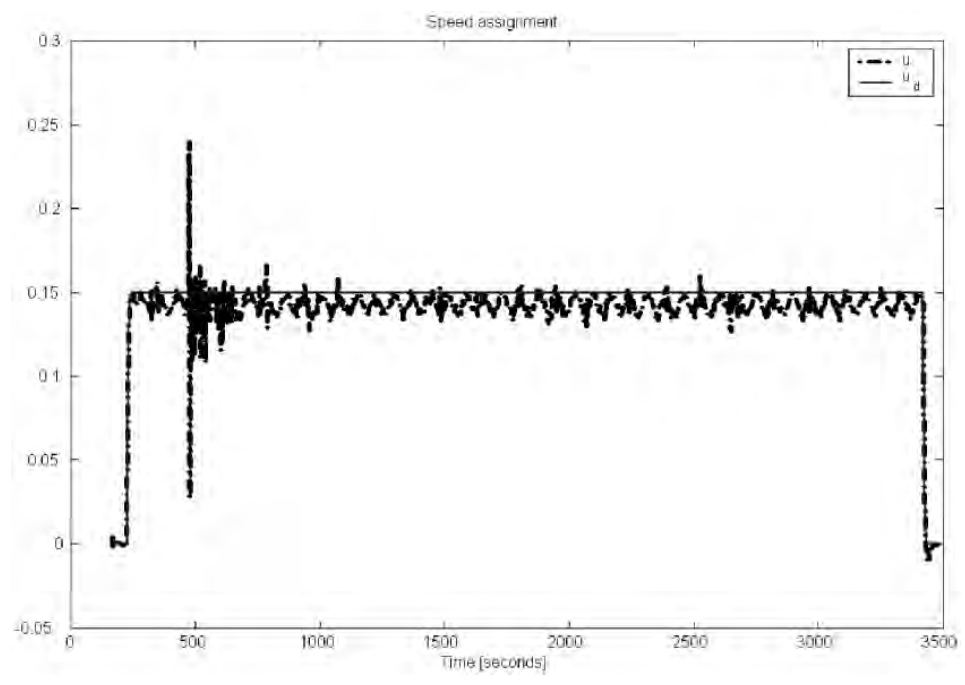

Figure 13. The desired and actual surge speed of CyberShip II for the full experiment. Notice the discrepancies around $t \approx 500 \mathrm{~s}$ which resulted from position measurement outages.
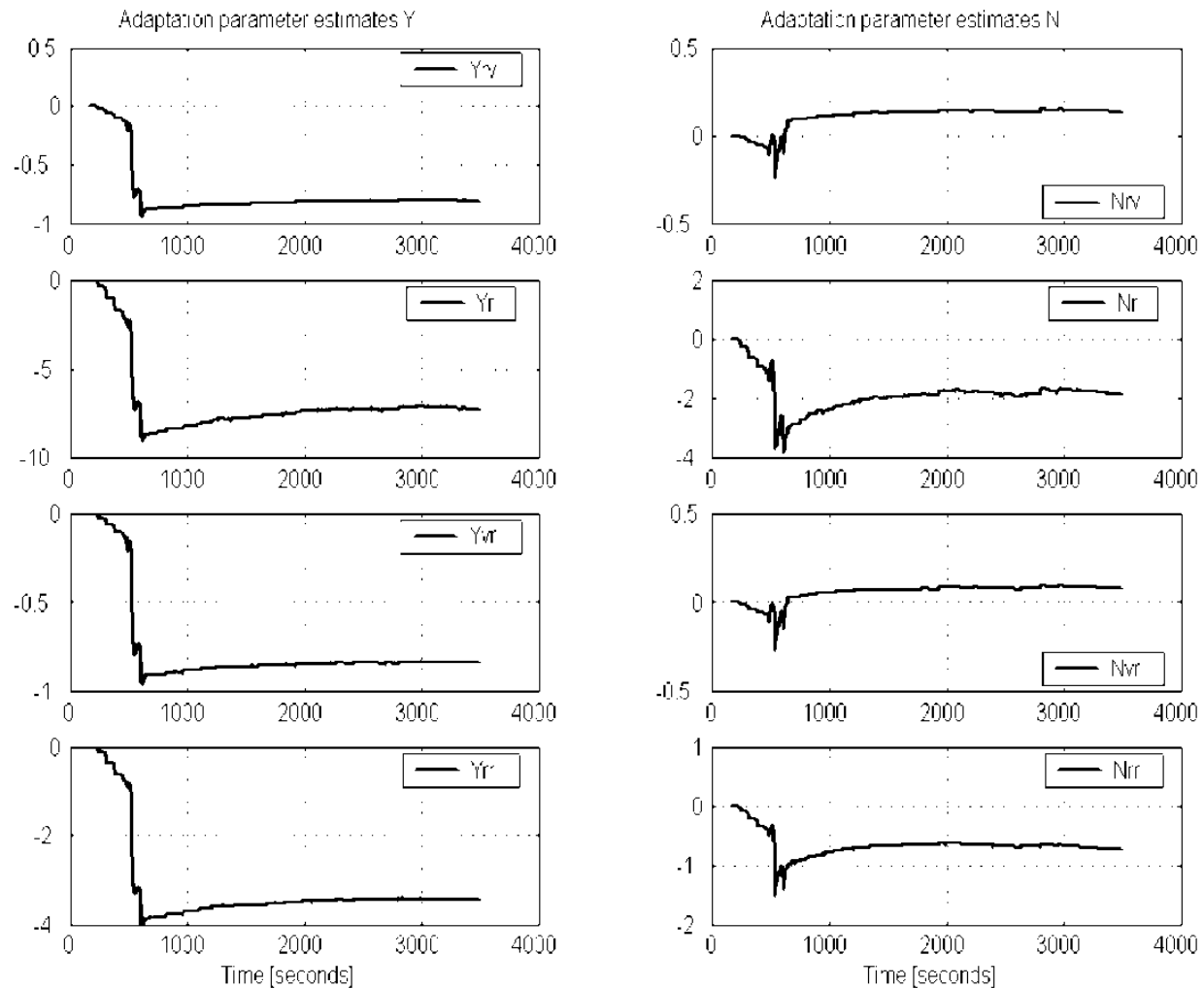

Figure 14. Adaptive parameter estimates $\hat{\varphi}(t)$ in the free-running CyberShip II manoeuvering experiment. 
Table 4. Adaptively estimated parameters for CyberShip II

\begin{tabular}{llcr}
\hline$Y_{|r| v}$ & -0.805 & $N_{|r| v}$ & 0.130 \\
$Y_{r}$ & -7.250 & $N_{r}$ & -1.900 \\
$Y_{|v| r}$ & -0.845 & $N_{|v| r}$ & 0.080 \\
$Y_{|r| r}$ & -3.450 & $N_{|r| r}$ & -0.750 \\
\hline
\end{tabular}

Table 5. Standard deviations for CyberShip II in the free-running manoeuvering experiment

\begin{tabular}{ccccc}
\hline$u_{d}[\mathrm{~m} / \mathrm{s}]$ & $z_{11}[\mathrm{~m}]$ & $z_{12}[\mathrm{~m}]$ & $z_{13}[\mathrm{deg}]$ & $u-u_{d}[\mathrm{~m} / \mathrm{s}]$ \\
\hline 0.15 & 0.0350 & 0.0226 & 2.623 & 0.0080 \\
\hline
\end{tabular}

This corresponds to an accuracy of $1.58 \mathrm{~m}$ for the full scale ship having a breadth of $20.3 \mathrm{~m}$.

\section{Conclusion}

We have presented a modeling, identification, and control design for the task of manoeuvering a ship along desired paths. The identification and adaptive manoeuvering procedure with experiments have provided numerical values for all parameters in the nonlinear ship model for CyberShip II. It was the intention of the authors to quantify such a model and share it with the marine control research community for use in simulations and case studies. Material from a rich variety of references have been used to describe the model, its difficulties and possible simplifications.

System identification procedures, using a towing carriage in the Marine Cybernetics Laboratory in Trondheim, Norway, were performed where the model ship CyberShip II was towed at many different velocities and the average towing forces were recorded. For zero acceleration and zero input forces these measurements are directly related to the drag of the ship hull. These measurements were accurately fitted to a nonlinear damping model of the ship for pure surge and sway motions. Knowing these nominal models, the same towing tests were repeated, with the thrusters and rudders activated, to find the actuator models. After these tests, eight damping parameters related to the yaw rate of the ship were still unknown. To find these, an adaptive manoeuvering control law was implemented and experimentally tested. The estimates of the unknown parameters in this experiment were assumed to be close to the true values and therefore adopted as the remaining numerical values.

In summary, this design with experimental testing has provided a complete manoeuvering model with numerical values for CyberShip II. The accuracy of the obtained parameters are believed to be close to the true values (as far as this is possible to quantify for a nonlinear ship model that still is a mere simplification of the real world). Nonetheless, the free-running manoeuvering experiment using a robust adaptive control law showed that accurate manoeuvering along desired paths is very much achievable in presence of modeling uncertainties and exogenous disturbances.

\section{Acknowledgement}

We wish to thank Dag Abel Sveen for his support and patience in the towing experiments. Supported by the Norwegian Research Council through the Strategic University Program on Marine Control. 


\section{References}

Aвкоwitz, M. A. (1964). Lectures on ship hydrodynamics—-steering and manoeuvrability, Technical report Hy-5, Hydro- and Aerodynamics Laboratory, Lyngby, Denmark.

Anderson, B. D. O., Bitmead, R. R., Johnson, JR., C. R., Kokotović, P. V., Kosut, R. L., Mareels, I. M. Y., Praly, L. \& Riedle, B. D. (1986). Stability of adaptive systems, MIT Press Series in Signal Processing, Optimization, and Control, 8, The MIT Press, Cambridge, MA, USA. Passivity and averaging systems.

Blanke, M. (1981). Ship Propulsion Losses Related to Automated Steering and Prime Mover Control, Phd thesis, Technical Univ. of Denmark, Lyngby, Denmark.

Clarke, D. (2003). The foundations of steering and manoeuvring, Proc. IFAC Conf. Manoeuvering and Control of Marine Crafts, IFAC, Girona, Spain.

Davidson, K. S. M. \& Schiff, L. I. (1946). Turning and course keeping qualities, Trans. Soc. of Nav. Architects Marine Eng. 54: 152-200.

Faltinsen, O. M. (1990). Sea Loads on Ships and Offshore Structures, Cambridge University Press.

Fedyaevsky, K. K. \& Sobolev, G. V. (1963). Control and Stability in Ship Design, State Union Shipbuilding Publishing House, Leningrad. 26

Fossen, T. (1994). Guidance and Control of Ocean Vehicles, John Wiley \& Sons Ltd, England.

Fossen, T. I. (2002). Marine Control Systems: Guidance, Navigation, and Control of Ships, Rigs and Underwater Vehicles, Marine Cybernetics, Trondheim, Norway.

Fossen, T. I., Breivik, M. \& SkJETne, R. (2003). Line-of-sight path following of underactuated marine craft, Proc. IFAC Conf. Manoeuvering and Control of Marine Crafts, IFAC, Girona, Spain, pp. 244.249.

Fossen, T. I. \& Strand, J. P. (1999). Passive nonlinear observer design for ships using lyapunov methods: full-scale experiments with a supply vessel, Automatica 35(1): 3-16.

HolzHÜTER, T. (1997). LQG approach for the high-precision track control of ships, IEE Proc. Control Theory Appl. 144(2): 121-127.

Johansen, T. A., Fuglseth, T. P., Tøndel, P. \& Fossen, T. I. (2003). Optimal constrained control allocation in marine vessels with rudders, Proc. IFAC Conf. Manoeuvering and Control of Marine Crafts, IFAC, Girona, Spain.

Krstić, M., Kanellakopoulos, I. \& Kokotović, P. (1995). Nonlinear and Adaptive Control Design, John Wiley \& Sons Ltd, New York.

Lefeber, E., Pettersen, K. Y. \& NiJMeijer, H. (2003). Tracking control of an underactuated ship, IEEE Trans. Contr. Sys. Tech. 11(1): 52-61.

LewIs, E. V. (ED.) (1988). Principles of Naval Architecture: Volume II-Resistance, Propulsion, and Vibration, Vol. 2, 2 edn, Soc. Naval Architects \& Marine Engineers.

Lindegand, K.-P. (2003). Acceleration Feedback in Dynamic Positioning, Phd thesis, Norwegian University of Science \& Technology, Trondheim, Norway.

LindegaARD, K.-P. \& Fossen, T. I. (2003). Fuel efficient rudder and propeller control allocation for marine craft: Experiments with a model ship, IEEE Trans. Contr. Sys. Tech. 11(6).

Meriam, J. L. \& Kraige, L. G. (1993). Engineering Mechanics: Dynamics, Vol. 2, 3 edn, John Wiley \& Sons Ltd, New York.

Newman, J. N. (1999). Marine Hydrodynamics, 9 edn, The MIT Press, Cambridge, Massachusetts.

Nomoto, K., Taguchi, T., Honda, K. \& Hirano, S. (1957). On the steering qualities of ships, Technical report 4, Int. Shipbuilding Progress.

Norrbin, N. H. (1970). Theory and observation on the use of a mathematical model for ship manoeuvering in deep and confined waters, 8th Symp. Naval Hydrodynamics, Pasadena, California, USA.

SkJETnE, R. \& Fossen, T. (2004). On integral control in backstepping: Analysis of different techniques, Proc. American Control Conf., AACC, Boston, USA. Submitted.

SkJetne, R., Fossen, T. I. \& Kokotović, P. V. (2004a). Adaptive output maneuvering with experiments for a model ship in a marine control laboratory, Automatica. Submitted Nov. 12, 2003.

SkJetne, R., Fossen, T. I. \& Kokotović, P. V. (2004b). Robust output maneuvering for a class of nonlinear systems, Automatica 40(3): 373-383.

Sørensen, A. (2002). Kompendium: Marine Cybernetics-Modelling and Control, Marine Technology Centre, Trondheim, Norway. 
Sørensen, A. J., Sagatun, S. I. \& Fossen, T. I. (1996). Design of a dynamic positioning system using model-based control, Control Eng. Practice 4(3): 359-368.

Strand, J. P. (1999). Nonlinear Position Control Systems Design for Marine Vessels, Phd thesis, Norwegian University of Science \& Technology, Trondheim, Norway. The Society of Naval Architects and Marine Engineers (1950). Nomenaclature for treating the motion of a submerged body through a fluid, Technical and Research Bulletin No. 1-5. 